\title{
Nuclear activity in galaxy pairs: a spectroscopic analysis of 48 UZC-BGPs ${ }^{\star}$
}

\author{
P. Focardi ${ }^{1}$, V. Zitelli ${ }^{2}$, and S. Marinoni ${ }^{1,3}$ \\ 1 Dipartimento di Astronomia, Universitá di Bologna, Italy \\ e-mail: paola. focardi@unibo.it \\ 2 INAF - OABO, Via Ranzani 1, 40127 Bologna, Italy \\ ${ }^{3}$ Fundacion Galileo Galilei \& TNG, PO Box 565 S/C de la Palma, Tenerife, Spain
}

Received 26 July 2007 / Accepted 8 March 2008

ABSTRACT

\begin{abstract}
Context. The role played by interaction on galaxy formation and evolution continues to be debated. Several questions remain open, among them whether, and to what extent, galaxy interaction induce nuclear activity, as theoretical predictions, so far, have not been adequately supported by observations. Part of the uncertainty affecting the observational results is likely to be due to the limited sizes and the inhomogeneity of the samples.

Aims. Galaxy pairs are ideal sites in which to investigate the role of interaction on nuclear activity, since the proximity, in redshift and in projected separation, between members make interaction and encounters highly probable. For this reason we have undertaken a spectroscopic survey of a large homogeneous sample of galaxy pairs (UZC-BGP) selected applying an objective neighbour search algorithm to a $3 \mathrm{D}$ galaxy catalog (UZC).

Methods. We present the results of the nuclear spectral classification, performed using standard diagnostic diagrams, of 48 UZC-BGPs, which represents more than half of the whole sample and has an excellent morphological match with it.

Results. The fraction of emission line galaxies in our pair sample is large, especially among spirals where it reaches $84 \%$ and $95 \%$, for early and late spirals. Star Burst (SB) is the most frequent type of nuclear activity encountered (30\% of galaxies), while AGNs (Active Galactic Nuclei) make only 19\%. The fractions increase to $45 \%$ and $22 \%$ when considering only spirals. Late spirals are characterized by both an unusual increase (35\%) of AGN activity and high luminosity ( $44 \%$ have $M_{B}<-20.0+5 \log h$ ). LLAGNs (Low Luminosity AGNs) are only $8 \%$ of the total number of galaxies, but this kind of activity could be present in another $10 \%$ of the galaxies (LLAGN candidates). If confirmed, these candidates would make LLAGNs constitute a significant fraction of the whole AGN (LLAGN + AGN) population, and raise the AGN population as a whole to 37\%. Absorption line galaxies reside mostly (61\%) in S0 galaxies and display the lowest $B$ luminosity in the sample; only $18 \%$ of them have $M_{B}<-20+5 \log h$, but together with LLAGNs (candidates included) they are the most massive galaxies in the sample. Intense-SB nuclei are found in galaxy pairs with galaxy-galaxy projected separation of up to $160 \mathrm{~h}^{-1} \mathrm{kpc}$ suggesting that in bright isolated galaxy pairs interaction may be at work and effective up to that distance.

Conclusions. AGNs are characterized by an advanced morphological type while the SB phenomenon occurs with the same frequency in early and late spirals. Whether and how these unusual characteristics relate to the pair environment needs to be further investigated. LLAGNs and LLAGN candidates do not always show similar properties; the former are more luminous in $B$, richer in early-type (E-S0s) galaxies, and half of them are hosted in galaxies showing visible signs of interaction with fainter companions. This last finding suggests that minor interactions might be a driving mechanism for a fraction of LLAGNs. The differences between LLAGNs and LLAGN candidates might confirm the heterogeneous nature of this class of objects.
\end{abstract}

Key words. galaxies: active - galaxies: interactions

\section{Introduction}

The role played by interactions; on galaxy formation and evolution remains hotly debated, involving both the "far" and "local" universe. Hierarchical models of galaxy formation invoke a regular occurrence of interaction and merging phenomena, which are expected to increase with redshift (Governato et al. 1999; Gottlober et al. 2001) and affect morphologies, gas distribution and the population of galaxies (Dubinski et al. 1996; Mihos \& Hernquist 1996). Evolution of the merging rate with $z$ has been estimated using close galaxy pairs, but results are conflicting (Zepf \& Koo 1989; Carlberg et al. 1994; Le Fevre et al. 2000; Bundy et al. 2000), which is not unexpected due to differences in sample depths, observational techniques and selection

\footnotetext{
* Based on observations obtained with the Cassini telescope in Loiano (BO).
}

criteria (Patton et al. 2000). Interaction and mergers are less frequent in the "local" universe, but can be analysed in more detail. Nearby galaxy systems can be investigated at faint luminosity, on a wide spatial scale and with a better knowledge of the surrounding environment. However, even "locally" theoretical expectations have not found adequate support from observational data. Galaxy interaction should be extremely effective in redistributing large amounts of material towards the galaxy central regions, giving rise, in this way, to violent bursts of star formation (Barnes \& Hernquist 1991). Tidal interaction between galaxies is expected to induce instabilities in the discs, able to generate galaxy bar formation, which, producing an inflow of gas towards the galaxy central regions, may even activate the AGN phenomenon (Noguchi 1988; Barnes \& Hernquist 1991). However, observations concerning the amount and level of nuclear activity in nearby interacting systems so far have produced 
conflicting results neither able to confirm nor to definitely rule out theoretical expectations. In fact, eventhough, starting with Larson \& Tinsley (1988), there has been growing evidence of an increase in star formation in interacting galaxy systems (e.g. Kennicutt \& Keel 1984; Kennicutt et al. 1987; Keel 1993, 1996; Donzelli \& Pastoriza 1997; Barton et al. 2000), a one-to-one correlation between galaxy-galaxy interaction and star formation remains unclear. Such a correlation holds only for an extremely limited number of objects (ULIRGs, Sanders \& Mirabel 1996) which show a fraction of interacting galaxies nearly close to $100 \%$ (Sanders et al. 1988; Borne et al. 1999), while there are several interacting systems with no sign of star formation. The situation becomes even more complex and controversial for the so-called AGN-interaction paradigm for which conflicting results have been given so far (Dahari 1985; Keel et al. 1985; Fuentes-Williams \& Stocke 1988; Rafanelli et al. 1995; MacKenty 1989; Kelm et al. 1998; De Robertis et al. 1998; Schmitt 2001; Kelm et al. 2004). However a large part of this contradiction is likely to be due to inhomogeneities among the analyzed samples which are often small, have been selected by different methods and criteria, and may be biased towards or against certain kind of systems.

Galaxy pairs are ideal sites to investigate the role of interaction on nuclear activity since proximity in redshift and in projected separation make interaction and encounters between member galaxies highly probable. The recent availability of a large and complete nearby 3D galaxy catalog (UZC, Falco et al. 1999) has made it feasible to select a volume-limited sample of 89 Bright Galaxy Pairs (UZC-BGP, Focardi et al. 2006, hereafter Paper I) which does not suffer from velocity/distance biases or contamination by projection effects. At variance with previous available nearby pair samples (KPG, Karachentsev 1972; RR, Reduzzi \& Rampazzo 1995), the first selected visually from the POSS plates, the second applying KPG criteria to the ESO-LV catalog (Lauberts \& Valentijn 1989), the UZC-BGP sample has been selected by means of an objective neighbour search algorithm (Focardi \& Kelm 2002) applied to the UZC catalog; it is thus complete, homogeneous and contains pairs which are already close in $3 \mathrm{D}$ space.

The analysis of UZC-BGP, based on available data (Paper I), has allowed us to show that ellipticals are extremely rare and underluminous (in $B$ ), while late spirals $(>\mathrm{Sc})$ are overluminous. This finding confirms previous claims (Kelm \& Focardi 2004) linking the formation of bright ellipticals to group/cluster environment and suggest that galaxy-galaxy interactions might be responsible for the blue luminosity enhancement of disk galaxies through SF phenomena. This last suggestion found support in the strong FIR emission displayed by a significant fraction of early spirals, mostly belonging to interacting pairs.

Very little is available (see Paper I) concerning nuclear activity type in UZC-BGP; we have thus undertaken a spectroscopic survey of the sample. The survey is currently still ongoing (now $85 \%$ complete). In this paper we present results and analysis for 48 UZC-BGPs which constitute more than half of the whole sample and have an excellent morphological match with it.

The sample is presented in Sect. 2; in Sect. 3 we show the results of our nuclear activity classification based on standard diagnostic diagrams; in Sect. 4 we analyze and compare the characteristics of galaxies having different nuclear activity type; in Sect. 5 we look for a possible link between nuclear activity and interaction strength. The conclusions are drawn in Sect. 6. As in Paper I, a Hubble constant of $H_{0}=100 \mathrm{hm} \mathrm{s}^{-1} \mathrm{Mpc}^{-1}$ is assumed throughout.

\section{The sample}

The sample contains 48 galaxy pairs, which represent more than half $(54 \%)$ of UZC-BGP sample. The latter is a volume-limited sample of galaxy pairs selected from the UZC catalog applying an adapted version of the neighbour search algorithm of Focardi \& Kelm (2002). The environment of each UZC galaxy with $M_{\mathrm{Zw}} \leq-18.87+5 \log h, v_{\mathrm{r}} \in[2500-7500] \mathrm{km} \mathrm{s}^{-1}$ and $\left|b^{\mathrm{II}}\right| \geq$ $30^{0}$; has been explored in a surronding area characterized by two projected dimensions $\left(r_{\mathrm{p}}=200 h^{-1} \mathrm{kpc}\right.$ and $\left.R_{\mathrm{p}}=1 h^{-1} \mathrm{Mpc}\right)$ and a radial velocity "distance" $\left(\left|\Delta v_{\mathrm{r}}\right|=1000 \mathrm{~km} \mathrm{~s}^{-1}\right)$. Galaxies having only one bright neigbour $\left(M_{\mathrm{Zw}_{\mathrm{w}}} \leq-18.87+5 \log h\right)$ within $r_{\mathrm{p}}$ and $\left|\Delta v_{\mathrm{r}}\right|$ and no other ones up to $R_{\mathrm{p}}$ (and within $\left.\left|\Delta v_{\mathrm{r}}\right|\right)$ were included in the UZC-BGP sample. The adopted value for $r_{\mathrm{p}}$ (galaxy-galaxy projected distance) accounts for possible large haloes linked to bright galaxies (Bahcall et al. 1995; Zaritsky et al. 1997). The value for $\left|\Delta v_{\mathrm{r}}\right|$ is large enough to not induce an artificial cut in the relative velocities of galaxies in pairs (within $r_{\mathrm{p}}$ ) and to prevent contamination by galaxy groups/clusters (up to $R_{\mathrm{p}}$ ). The value for $R_{\mathrm{p}}$ (large scale isolation radius) was chosen to ensure the absence of luminous companions on group/cluster typical scales. The lower limit in radial velocity was fixed to reduce distance uncertainities due to peculiar motions and to prevent contamination by the Virgo complex, while the upper limit was set to guarantee sampling of the UZC luminosity function just below $L^{*}$ (Cuesta-Bolao \& Serna 2003). A limit in $\left|b^{\mathrm{II}}\right|$ was imposed to minimize the effects of galactic absorption.

Unlike other galaxy pair samples selected from 3D catalogs (Barton et al. 2000; Alonso et al. 2004) that are magnitude limited and contain pairs belonging to any kind of large scale environment, UZC-BGP is particularly suited to investigate the mutual effects of two bright close companions, isolated on the typical group/cluster scale. Minor companions, which might have failed to enter either the UZC $\left(m_{\mathrm{Zw}} \leq 15.5\right)$ or UZC-BGP $\left(M_{\mathrm{Zw}} \leq-18.87+5 \log h\right)$ luminosity limit, could be present in the local (within $r_{\mathrm{p}}$ ) or distant (within $R_{\mathrm{p}}$ ) environment but are not expected to play a role comparable to that of the two massive galaxies in the pair. UZC-BGP galaxies are rather bright and, since luminosity relates to mass although not in an obvious way, are rather massive ${ }^{1}$ too. (Further details on the UZC-BGP sample can be found in Paper I).

Following Karachentsev (1972), galaxy pairs can be classified on the basis of their morphology in E+E, S+S and E+S pairs. E+E pairs contain only early-type galaxies $(\mathrm{E}+\mathrm{S} 0 \mathrm{~s}), \mathrm{S}+\mathrm{S}$ pairs only spirals and $\mathrm{E}+\mathrm{S}$ pairs both types. The sample we present here is a fair representation of the whole UZC-BGP as it contains $6 \mathrm{E}+\mathrm{E}(12 \%), 23 \mathrm{~S}+\mathrm{S}(48 \%)$ and $19 \mathrm{E}+\mathrm{S}(40 \%)$ pairs, which are remarkably similar to the ones of the whole UZC-BGP sample $(13 \%, 48 \%$ and $39 \%)$.

Two dimensional long slit spectra have been acquired with BFOSC (the Bologna Faint Object Spectrograph and Camera) at the $152 \mathrm{~cm}$ telescope (of Bologna University) in Loiano (Italy). Spectral coverage is $4000-8500 \AA$ with an average resolution of about $4 \AA$. The slit was positioned over the nucleus of each galaxy and its width was set either at $2^{\prime \prime}$ or at $2.5^{\prime \prime}$ (depending on seeing conditions), which corresponds to the galaxy nuclear region (about $500 \mathrm{~h}^{-1} \mathrm{pc}$ at the average redshift of our sample). The data reduction was performed with IRAF. After

\footnotetext{
1 A rough estimate of the minimum mass $\left(M_{\min }\right)$ of UZC-BGP galaxies can be derived from their minimum luminosity $L_{B} \sim 5.5 \times 10^{9} h^{-2} L_{\odot}$ and assuming, for them, an average $M / L$ of $5 h^{-1}$, this gives $M_{\min } \sim$ $2.8 \times 10^{10} h^{-1} M_{\odot}$.
} 
Table 1. The E+E pairs.

\begin{tabular}{lrlll}
\hline \hline Name & UZC-BGP & Type & $T$ & Emission lines \\
\hline NGC 2991 & 23A & S0 & -2.1 & - \\
NGC 2994 & 23B & S0 & -2.0 & - \\
NGC 3567 & 35A & S0 & -2.1 & - \\
CGCG 039-055 & 35B & E & -2.7 & - \\
CGCG 160-030 & 51A & S0/a & -1.4 & - \\
CGCG 160-036 & 51B & E & -4.3 & {$[\mathrm{NII}]_{6583}$} \\
CGCG 102-023 & 58A & S0 & -1.7 & - \\
CGCG 102-024 & 58B & S0 & -1.8 & - \\
IC 999 & 63A & S0 & -1.9 & - \\
IC 1000 & 63B & S0 & -2.0 & - \\
NGC 6018 & 77A & SB0/a & -1.2 & {$[\mathrm{NII}]_{6548}, \mathrm{H}_{\alpha},[\mathrm{NII}]_{6583},[\mathrm{SII}]_{6717},[\mathrm{SII}]_{6731}$} \\
NGC 6021 & 77B & E & -4.0 & {$[\mathrm{NII}]_{6583}$} \\
\hline
\end{tabular}

standard CCD (flat field and bias) correction, we extracted the spectra, calibrated them in wavelength, identified the emission lines and measured their $E W$. Spectral extraction was limited to the 4-5 central pixels, corresponding to $2^{\prime \prime}-2.5^{\prime \prime}$ at the detector scale. We set the continuum level in the close neighborhood of each emission line (on both sides) and when the emission $\left(\mathrm{H}_{\alpha}\right.$ and/or $\mathrm{H}_{\beta}$ ) was affected by the presence of an underlaying strong absorption line, we set the continuum at the bottom of the emission line, to minimize the effect of the absorption.

Galaxies in $\mathrm{E}+\mathrm{E}, \mathrm{S}+\mathrm{S}$ and $\mathrm{E}+\mathrm{S}$ pairs are listed respectively in Tables $1-3$. In each table we give the galaxy name, (Col. 1), the UZC-BGP identifier (Col. 2), morphological classification (type) and type code ( $T$ ) (both from LEDA, Cols. 3 and 4), and emission lines identified in each spectrum, if any (Col. 5). We have identified only lines with $S / N \geq 5$; very few lines, however, are characterized by such a low signal, and the vast majority has, on average, $S / N \geq 50$. Morphology is very well defined for $86 \%$ (83/96) of the galaxies, is less defined for 13 galaxies and, in these last cases the morphological classification (Col. 3) is followed by a question mark.

Inspection of Tables 1 to 3 provides evidence that emission line galaxies are extremely rare in $\mathrm{E}+\mathrm{E}$, overabundant in $\mathrm{S}+\mathrm{S}$ and rather frequent (more than half of the galaxies) in $\mathrm{E}+\mathrm{S}$ pairs. There are 3 galaxies with emission lines in the E+E pairs representing $25 \%$ of the total number of galaxies, 39 emission line galaxies in the $\mathrm{S}+\mathrm{S}(85 \%)$ and 26 in the $\mathrm{E}+\mathrm{S}(68 \%)$ pairs. The different fractions are obviously related to the different morphological content of the pairs and this is clearly illustrated in Fig. 1, whose 4 panels show the morphological distribution of galaxies in the whole sample (upper left), E+E (upper right), $S+S$ (lower left) and $\mathrm{E}+\mathrm{S}$ (lower right) pairs. Morphology is represented, on the $x$ axis, by the type code $T$ (Col. 4 in each table), which is a numerical parametrization of the morphological type, introduced by de Vaucouleurs \& de Vaucouleurs (1964). According to this parametrization, early-type galaxies ( $\mathrm{E}$ and $\mathrm{S} 0$ s) have $T<0$ (E in general $T<-3$ ), while late spirals ( $>$ Sbc) have, on average, $T \geq 4$. The increase in emission line galaxies with morphology is clearly evident in all pair samples containing spiral galaxies (panels 1, 3 and 4 of Fig. 1) and especially in panels 1 and 4 which show the more frequent occurrence of emission features in spiral than in E-S0 galaxies.

The much higher occurrance of emission line galaxies among spirals than among early-type (E-S0s) galaxies is not unexpected as emission features occur more frequently in gas rich than in gas poor galaxies; however the two lower panels of Fig. 1 show that our sample is characterized by a morphological content which is never more advanced than $T=6$ (corresponding roughly to Sc galaxies). The frequency of emission lines among spirals attains the maximum value for morphologies more advanced than Sc as the the ratio of the current SFR (Star Formation Rate) to the average past one increases from about 0.01 in Sa to 1 in Sc-Irr galaxies (Kennicutt et al. 1994). The large number of emission lines occurring among early spirals in our sample might thus be partly induced by interaction.

The two lower panels of Fig. 1 show clearly that the fraction of emission galaxies is much lower in $\mathrm{E}+\mathrm{S}$ than in $\mathrm{S}+\mathrm{S}$ pairs simply because of the large number of early-type (E-S0s) galaxies in $\mathrm{E}+\mathrm{S}$ pairs. In this last sample, 11 of the 12 galaxies with only absorption lines in their spectrum are early-type galaxies, implying that the fractions of early-type galaxies and spirals with emission lines are $42 \%(8 / 19)$ and 95\% (18/19) respectively. If we compare the last fraction with the fraction $(85 \%)$ of emission line galaxies in $\mathrm{S}+\mathrm{S}$ we see that both $\mathrm{E}+\mathrm{S}$ and $\mathrm{S}+\mathrm{S}$ pairs have an equally high probability of hosting an emission line spiral.

Figure 2 shows the absolute magnitude $\left(M_{B}-5 \log h\right)$ distribution of ellipticals ( $T<-3$, upper left), S0s $(-3 \leq T<0$, upper right), and early $(0 \leq T<4$, lower left $)$ and late $(T \geq 4$, lower right) spirals. ( $M_{B}$ has been derived from $B$ which is available in LEDA, Paturel et al. 2003, for 95/96 galaxies of our sample.) In analogy with Fig. 1 the continuous distribution refers to the whole sample, the hatched one to galaxies with emission lines. The lower panels of Fig. 2 reveal the extremely large fraction of emission line galaxies in early and late spirals: $84 \%$ (37/44) and 95\% (19/20), respectively. Curiously, ellipticals (upper left) also appear often $(67 \%)$ in emission line galaxies, but the statistics are too low and do not allow us to draw definitive conclusions on this point. S0 galaxies, instead, are abundant in our sample (22/96) and appear to host a much more limited fraction (23\%) of emission line galaxies. From Fig. 2 we see that our sample is numerically dominated by early spirals (47\%), has an almost equal content of late spirals (21\%) and S0s (23\%) and contains a limited fraction $(9 \%)$ of ellipticals. In terms of luminosity $\left(M_{B}\right)$ S0s are characterized by more "faint" galaxies; about half of them $(12 / 22,55 \%)$ have $M_{B} \geq-19.5+5 \log h$. There are no ellipticals with $M_{B}$ larger than that value, while early and late spirals (with $M_{B} \geq-19.5+5 \log h$ ) represent, respectively, 25\% and $15 \%$ of each population. Further investigation is required to establish whether the population of low luminosity SOs is typical of bright galaxy pairs and whether the lack of emission features in these galaxies is related to their low luminosity.

Figure 3 shows the distribution of galaxy-galaxy projected distance $\left(r_{\mathrm{p}}\right.$, upper panels) and velocity separation $\left(\left|\Delta v_{\mathrm{r}}\right|\right.$, lower panels) for $\mathrm{E}+\mathrm{E}, \mathrm{S}+\mathrm{S}$ and $\mathrm{E}+\mathrm{S}$ pairs. The continuous 
Table 2. The $S+S$ pairs.

\begin{tabular}{|c|c|c|c|c|}
\hline Name & UZC-BGP & Type & $T$ & Emission lines \\
\hline NGC 23 & $2 \mathrm{~A}$ & $\mathrm{SBa}$ & 1.2 & {$[\mathrm{OII}]_{3727}, \mathrm{H}_{\beta},[\mathrm{OIII}]_{4959},[\mathrm{OIII}]_{5007},[\mathrm{OI}]_{6300},[\mathrm{NII}]_{6548}, \mathrm{H}_{\alpha},[\mathrm{NII}]_{6583},[\mathrm{SII}]_{6717},[\mathrm{SII}]_{6731}$} \\
\hline NGC 26 & 2B & Sab & 2.4 & {$[\mathrm{NII}]_{6583}$} \\
\hline NGC 800 & $8 \mathrm{~A}$ & $\mathrm{Sc}$ & 5.3 & - \\
\hline NGC 799 & 8B & $\mathrm{SBa}$ & 1.1 & {$[\mathrm{NII}]_{6548}, \mathrm{H}_{\alpha},[\mathrm{NII}]_{6548},[\mathrm{SII}]_{6717},[\mathrm{SII}]_{6731}$} \\
\hline NGC 871 & 9A & $\mathrm{SBc}$ & 4.6 & {$[\mathrm{OII}]_{3727}, \mathrm{H}_{\beta},[\mathrm{OIII}]_{4959},[\mathrm{OIII}]_{5007},[\mathrm{OI}]_{6300},[\mathrm{NII}]_{6548}, \mathrm{H}_{\alpha},[\mathrm{NII}]_{6583},[\mathrm{SII}]_{6717},[\mathrm{SII}]_{6731}, \mathrm{HeI}_{7065}$} \\
\hline NGC 877 & 9B & $\mathrm{SBc}$ & 4.8 & {$[\mathrm{NII}]_{6548}, \mathrm{H}_{\alpha},[\mathrm{NII}]_{6583},[\mathrm{SII}]_{6717},[\mathrm{SII}]_{6731}$} \\
\hline UGC 4074 & $17 \mathrm{~A}$ & $\mathrm{Sc}$ & 5.9 & $\mathrm{H}_{\alpha}$ \\
\hline CGCG 262-048 & 17B & $\mathrm{Sc}$ & 5.0 & {$[\mathrm{NII}]_{6548}, \mathrm{H}_{\alpha},[\mathrm{NII}]_{6583}$} \\
\hline CGCG 035-023 & $22 \mathrm{~A}$ & S? & 1.6 & $\mathrm{H}_{\beta},[\mathrm{OIII}]_{5007},[\mathrm{OI}]_{6300},[\mathrm{NII}]_{6548}, \mathrm{H}_{\alpha},[\mathrm{NII}]_{6583},[\mathrm{SII}]_{6717},[\mathrm{SII}]_{6731}$ \\
\hline CGCG 035-25 & 22B & S? & 2.8 & $\mathrm{H}_{\beta},[\mathrm{OIII}]_{5007},[\mathrm{NII}]_{6548}, \mathrm{H}_{\alpha},[\mathrm{NII}]_{6583},[\mathrm{SII}]_{6717},[\mathrm{SII}]_{6731}$ \\
\hline UGC 5241 & $24 \mathrm{~A}$ & Sbc & 4.4 & $\mathrm{H}_{\beta},[\mathrm{OIII}]_{4959},[\mathrm{OIII}]_{5007}, \mathrm{HeII}_{5412},[\mathrm{OI}]_{6300}, \mathrm{H}_{\alpha},[\mathrm{NII}]_{6583},[\mathrm{SII}]_{6717},[\mathrm{SII}]_{6731}$ \\
\hline CGCG 265-043 & 24B & Sbc & 4.3 & {$[\mathrm{NII}]_{6548}, \mathrm{H}_{\alpha},[\mathrm{NII}]_{6583}$} \\
\hline NGC 3303 & $28 \mathrm{~A}$ & $\mathrm{Sb}$ & 3.2 & {$[\mathrm{OII}]_{3727}, \mathrm{H}_{\beta},[\mathrm{OIII}]_{4959},[\mathrm{OIII}]_{5007},[\mathrm{OI}]_{6300},[\mathrm{NII}]_{6548}, \mathrm{H}_{\alpha},[\mathrm{SII}]_{6717},[\mathrm{SII}]_{6731}$} \\
\hline CGCG 094-098 & 28B & S? & 3.8 & {$[\mathrm{OII}]_{3727}, \mathrm{H}_{\beta},[\mathrm{OIII}]_{4959},[\mathrm{OIII}]_{5007},[\mathrm{OI}]_{6300},[\mathrm{NIII}]_{6548}, \mathrm{H} \alpha,[\mathrm{NII}]_{6583},[\mathrm{SII}]_{6717},[\mathrm{SII}]_{6731}$} \\
\hline MKN 725 & $29 \mathrm{~A}$ & $\mathrm{Sc}$ & 4.7 & $\mathrm{H}_{\beta},[\mathrm{OIII}]_{4959},[\mathrm{OIII}]_{5007}, \mathrm{H}_{\alpha},[\mathrm{NII}]_{6583},[\mathrm{SII}]_{6717},[\mathrm{SII}]_{6731}, \mathrm{HeI}_{7065}$ \\
\hline UGC 5822 & 29B & SBa & 1.0 & {$[\mathrm{OII}]_{3727}, \mathrm{H}_{\gamma}, \mathrm{H}_{\beta},[\mathrm{OIII}]_{4959},[\mathrm{OIII}]_{5007}, \mathrm{HeI}_{5876},[\mathrm{OII}]_{6300},[\mathrm{NII}]_{6548}, \mathrm{H}_{\alpha},[\mathrm{NII}]_{6583},[\mathrm{SII}]_{6717},[\mathrm{SII}]_{6731}$} \\
\hline UGC 6033 & $31 \mathrm{~A}$ & $\mathrm{Sb}$ & 3.0 & - \\
\hline CGCG 213-008 & 31B & S? & 0.7 & - \\
\hline UGC 6397 & $36 \mathrm{~A}$ & Sab & 2.0 & {$[\mathrm{NII}]_{6548}, \mathrm{H}_{\alpha},[\mathrm{NII}]_{6583}$} \\
\hline CGCG 185-053 & $36 \mathrm{~B}$ & Sab & 1.5 & {$[\mathrm{NII}]_{6548}, \mathrm{H}_{\alpha},[\mathrm{NII}]_{6583},[\mathrm{SII}]_{6717},[\mathrm{SII}]_{6731}$} \\
\hline NGC 3719 & $37 \mathrm{~A}$ & $\mathrm{Sbc}$ & 3.7 & {$[\mathrm{NII}]_{6548}, \mathrm{H}_{\alpha},[\mathrm{NII}]_{6583}$} \\
\hline NGC 3720 & 37B & $\mathrm{Sa}$ & 1.4 & $\mathrm{H}_{\alpha},[\mathrm{NII}]_{6583},[\mathrm{SII}]_{6717},[\mathrm{SII}]_{6731}$ \\
\hline UGC 7383 & $44 \mathrm{~A}$ & Sab & 2.0 & $\mathrm{H}_{\beta},[\mathrm{OIII}]_{5007}, \mathrm{H}_{\alpha},[\mathrm{NII}]_{6583}$ \\
\hline VCC 0395 & 44B & Sbc & 4.5 & {$[\mathrm{NII}]_{6548}, \mathrm{H}_{\alpha},[\mathrm{NII}]_{6583}$} \\
\hline IC 962 & $59 \mathrm{~A}$ & $\mathrm{Sa}$ & 0.6 & {$[\mathrm{NII}]_{6548}, \mathrm{H}_{\alpha},[\mathrm{NII}]_{6583}$} \\
\hline CGCG 074-014 & $59 \mathrm{~B}$ & Sab & 1.7 & {$[\mathrm{OIII}]_{4959},[\mathrm{OIII}]_{5007},[\mathrm{NII}]_{6548}, \mathrm{H}_{\alpha},[\mathrm{NII}]_{6583},[\mathrm{SII}]_{6717},[\mathrm{SII}]_{6731}$} \\
\hline Mrk 820 & $66 \mathrm{~A}$ & S0/a & 0.1 & {$[\mathrm{OII}]_{3727}, \mathrm{H}_{\beta},[\mathrm{OIII}]_{4959},[\mathrm{OIII}]_{5007},[\mathrm{NII}]_{6548}, \mathrm{H}_{\alpha},[\mathrm{NII}]_{6583},[\mathrm{SII}]_{6717},[\mathrm{SII}]_{6731}$} \\
\hline UGC 9464 & $66 \mathrm{~B}$ & S0/a & 0.0 & - \\
\hline IC 1075 & $68 \mathrm{~A}$ & $\mathrm{SBb}$ & 3.4 & {$[\mathrm{OII}]_{3727}, \mathrm{H}_{\beta},[\mathrm{OIII}]_{4959},[\mathrm{OIII}]_{5007}, \mathrm{HeI}_{5896},[\mathrm{OI}]_{6300},[\mathrm{NII}]_{6548}, \mathrm{H}_{\alpha},[\mathrm{NII}]_{6583},[\mathrm{SII}]_{6717},[\mathrm{SII}]_{6731}$} \\
\hline IC 1076 & 68B & $\mathrm{Sb}$ & 2.8 & {$[\mathrm{NII}]_{6548}, \mathrm{H}_{\alpha},[\mathrm{NII}]_{6583},[\mathrm{SII}]_{6717},[\mathrm{SII}]_{6731}$} \\
\hline NGC 5797 & 69A & $\mathrm{S} 0 / \mathrm{a}$ & 0.0 & - \\
\hline NGC 5804 & 69B & $\mathrm{SBb}$ & 3.1 & {$[\mathrm{OII}]_{3727}, \mathrm{H}_{\gamma}, \mathrm{H}_{\beta},[\mathrm{OIII}]_{4959},[\mathrm{OIII}]_{5007},[\mathrm{OI}]_{6300},[\mathrm{NII}]_{6548}, \mathrm{H}_{\alpha},[\mathrm{NII}]_{6583},[\mathrm{SII}]_{6717},[\mathrm{SII}]_{6731}$} \\
\hline NGC 5857 & $71 \mathrm{~A}$ & SBab & 2.4 & {$[\mathrm{NII}]_{6583}$} \\
\hline NGC 5859 & $71 \mathrm{~B}$ & $\mathrm{SBbc}$ & 4.0 & {$[\mathrm{NII}]_{6548}, \mathrm{H}_{\alpha},[\mathrm{NII}]_{6583},[\mathrm{SII}]_{6717},[\mathrm{SII}]_{6731}$} \\
\hline CGCG 354-023 & $74 \mathrm{~A}$ & S? & 2.8 & {$[\mathrm{OII}]_{3727}, \mathrm{H}_{\beta},[\mathrm{OIII}]_{4959},[\mathrm{OIII}]_{5007},[\mathrm{OI}]_{6300},[\mathrm{NII}]_{6548}, \mathrm{H}_{\alpha},[\mathrm{NII}]_{6583},[\mathrm{SII}]_{6717},[\mathrm{SII}]_{6731}$} \\
\hline CGCG 354-023 & $74 \mathrm{~B}$ & S? & 2.8 & {$[\mathrm{OII}]_{3727}, \mathrm{H}_{\beta},[\mathrm{OIII}]_{4959},[\mathrm{OIII}]_{5007},[\mathrm{OI}]_{6300},[\mathrm{NII}]_{6548}, \mathrm{H}_{\alpha},[\mathrm{NII}]_{6583},[\mathrm{SII}]_{6717},[\mathrm{SII}]_{6731}$} \\
\hline IC 4576 & $76 \mathrm{~A}$ & $\mathrm{~S}$ ? & 0.3 & - \\
\hline IC 4577 & 76B & S? & 1.0 & - \\
\hline NGC 6246 & $80 \mathrm{~A}$ & $\mathrm{SBb}$ & 3.1 & $\mathrm{H}_{\alpha}$ \\
\hline NGC 6246 A & $80 \mathrm{~B}$ & $\mathrm{SBc}$ & 5.1 & {$[\mathrm{NIII}]_{6548}, \mathrm{H}_{\alpha},[\mathrm{NII}]_{6583},[\mathrm{SII}]_{6717},[\mathrm{SII}]_{6731}$} \\
\hline CGCG 452-021 & $81 \mathrm{~A}$ & $\mathrm{Sc}$ & 5.8 & $\mathrm{H}_{\beta},[\mathrm{OIII}]_{4959},[\mathrm{OIII}]_{5007},[\mathrm{NII}]_{6548}, \mathrm{H}_{\alpha},[\mathrm{NII}]_{6583},[\mathrm{SII}]_{6717},[\mathrm{SII}]_{6731}$ \\
\hline UGC 12067 & $81 \mathrm{~B}$ & $\mathrm{Sa}$ & 1.0 & $\mathrm{H}_{\beta},[\mathrm{OIII}]_{4959},[\mathrm{OIII}]_{5007},[\mathrm{OI}]_{6300},[\mathrm{NII}]_{6548}, \mathrm{H}_{\alpha},[\mathrm{NII}]_{6583},[\mathrm{SII}]_{6717},[\mathrm{SII}]_{6731}$ \\
\hline IC 5242 & $82 \mathrm{~A}$ & $\mathrm{Sa}$ & 1.9 & {$[\mathrm{OIII}]_{3727}, \mathrm{H}_{\beta},[\mathrm{OIII}]_{5007},[\mathrm{NII}]_{6548}, \mathrm{H}_{\alpha},[\mathrm{NII}]_{6583},[\mathrm{SII}]_{6583},[\mathrm{SII}]_{6731}$} \\
\hline IC 5243 & $82 \mathrm{~B}$ & $\mathrm{Sbc}$ & 3.6 & $\mathrm{H}_{\alpha}$ \\
\hline Mrk 308 & $83 \mathrm{~A}$ & $\mathrm{~S} 0 / \mathrm{a}$ & 0.3 & $\begin{array}{l}{[\mathrm{OIII}]_{3727}, \mathrm{H}_{\gamma}, \mathrm{H}_{\beta},[\mathrm{OIII}]_{4959},[\mathrm{OIII}]_{5007}, \mathrm{HeI}_{5876},[\mathrm{OI}]_{6300},[\mathrm{OI}]_{6364},[\mathrm{NII}]_{6548}, \mathrm{H}_{\alpha},[\mathrm{NII}]_{6583},} \\
{[\mathrm{SII}]_{6717},[\mathrm{SII}]_{6731}, \mathrm{HeI}_{7065},[\mathrm{OII}]_{7325}}\end{array}$ \\
\hline KUG $2239+200 \mathrm{~A}$ & $83 \mathrm{~B}$ & Sbc & 4.4 & $\begin{array}{l}{[\mathrm{OIII}]_{3727}, \mathrm{H}_{\gamma}, \mathrm{H}_{\beta},[\mathrm{OIII}]_{4959},[\mathrm{OIII}]_{5007}, \mathrm{HeI}_{5876},[\mathrm{OI}]_{6300},[\mathrm{NII}]_{6548}, \mathrm{H}_{\alpha},[\mathrm{NII}]_{6583},[\mathrm{SII}]_{6717},} \\
{[\mathrm{SII}]_{6731}, \mathrm{HeI}_{7065}}\end{array}$ \\
\hline
\end{tabular}

distribution indicates the whole sample, the dashed distributions indicate pairs having at least one member with an emission line spectrum (double dash) and pairs in which both members have emission lines (single dash). The fraction of pairs having both members "active" is clearly larger in $\mathrm{S}+\mathrm{S}(78 \%, 18 / 23)$ than in $\mathrm{E}+\mathrm{S}(42 \%, 8 / 19)$. This difference is caused by the early-type galaxy content of the $\mathrm{E}+\mathrm{S}$ pairs. The fractions become similar (91\% and $95 \%$ ) when considering $\mathrm{S}+\mathrm{S}$ and $\mathrm{E}+\mathrm{S}$ pairs in which at least one member has an emission line spectrum.

Previous work (Barton et al. 2000; Alonso et al. 2004) has claimed an increase in emission line galaxies in pairs with decreasing member projected distances. In our sample the fraction of emission line galaxies in $\mathrm{E}+\mathrm{S}$ and $\mathrm{S}+\mathrm{S}$ pairs is so high, at all member distances, that we hardly see such an effect. We stress however that UZC-BGP is a volume limited sample of bright isolated galaxy pairs quite different from the magnitude limited samples of close pairs belonging to any kind of environment, as in the samples in which Barton et al. (2000) and Alonso et al. (2004) observed a distance effect. The very large fraction of emission line galaxies in our sample at all pair distances may indicate that in isolated pairs of luminous $\left(M_{\mathrm{Zw}} \leq-18.9+5 \log h\right)$ galaxy interaction is at work and is effective up to $200 \mathrm{~h}^{-1} \mathrm{kpc}$. Both Barton et al. (2000) and Alonso et al. (2004) find emission line enhancement on a much smaller scale $\left(\sim 30 h^{-1} \mathrm{kpc}\right)$. Their result is likely to indicate that when galaxy pairs are surrounded by companions of comparable luminosity, galaxy-galaxy interaction becomes effective only at very small distances. Our result indicates that fainter companions which may be present in 
Table 3. The E $+\mathrm{S}$ pairs.

\begin{tabular}{|c|c|c|c|c|}
\hline Name & UZC-BGP & Type & $T$ & Emission lines \\
\hline NGC 41 & $3 \mathrm{~A}$ & Sc & 5.0 & {$[\mathrm{OII}]_{3727},[\mathrm{NII}]_{6548}, \mathrm{H}_{\alpha},[\mathrm{NII}]_{6583},[\mathrm{SII}]_{6717},[\mathrm{SII}]_{6731}$} \\
\hline NGC 42 & 3B & E/S0 & -3.0 & - \\
\hline NGC 160 & $4 \mathrm{~A}$ & $\mathrm{~S} 0 / \mathrm{a}$ & -0.4 & - \\
\hline NGC 169 & 4B & Sab & 2.5 & $\mathrm{H}_{\alpha},[\mathrm{NII}]_{6583},[\mathrm{SII}]_{6731}$ \\
\hline NGC 192 & $5 \mathrm{~A}$ & $\mathrm{SBa}$ & 1.0 & $\mathrm{H}_{\beta},[\mathrm{OIII}]_{4959},[\mathrm{OIII}]_{5007},[\mathrm{NII}]_{6548}, \mathrm{H} \alpha,[\mathrm{NII}]_{6583},[\mathrm{SII}]_{6717},[\mathrm{SII}]_{6731}$ \\
\hline NGC 196 & 5B & SB0 & -1.8 & - \\
\hline NGC 997S & $10 \mathrm{~A}$ & E & -3.8 & {$[\mathrm{NII}]_{6548}, \mathrm{H}_{\alpha},[\mathrm{NIII}]_{6583}$} \\
\hline NGC 998 & 10B & S? & 2.4 & {$[\mathrm{NII}]_{6548}, \mathrm{H}_{\alpha},[\mathrm{NII}]_{6583}$} \\
\hline Mkn 1076 & $14 \mathrm{~A}$ & Sbc & 3.8 & $\begin{array}{l}{[\mathrm{OII}]_{3727}, \mathrm{HeI}_{4026}, \mathrm{H}_{\gamma}, \mathrm{HeII}_{4686} \mathrm{H}_{\beta},[\mathrm{OIII}]_{4959},[\mathrm{OIII}]_{5007},[\mathrm{OI}]_{6300},[\mathrm{NII}]_{6548}, \mathrm{H}_{\alpha},[\mathrm{NII}]_{6583},[\mathrm{SII}]_{6717},} \\
{[\mathrm{SII}]_{6731}}\end{array}$ \\
\hline CGCG 390-059 & 14B & E/S0 & -2.9 & - \\
\hline NGC 1587 & $16 \mathrm{~A}$ & E & -4.8 & - \\
\hline NGC 1589 & 16B & Sab & 2.3 & {$[\mathrm{NII}]_{6583}$} \\
\hline NGC 2528 & $18 \mathrm{~A}$ & $\mathrm{SBb}$ & 3.1 & $\mathrm{H}_{\gamma}, \mathrm{H}_{\beta},[\mathrm{NII}]_{6548}, \mathrm{H}_{\alpha},[\mathrm{NII}]_{6583},[\mathrm{SII}]_{6717},[\mathrm{SII}]_{6731}$ \\
\hline NGC 2524 & 18B & $\mathrm{S} 0 / \mathrm{a}$ & -0.2 & - \\
\hline NGC 2744 & $20 \mathrm{~A}$ & SBab & 2.1 & $\begin{array}{l}{[\mathrm{OII}]_{3727}, \mathrm{HeI}_{4026}, \mathrm{H}_{\delta}, \mathrm{H}_{\gamma}, \mathrm{H}_{\beta},[\mathrm{OIII}]_{4959},[\mathrm{OIII}]_{5007}, \mathrm{HeI}_{5876},[\mathrm{OI}]_{6300},[\mathrm{NII}]_{6548}, \mathrm{H}_{\alpha},[\mathrm{NII}]_{6583},} \\
{[\mathrm{SII}]_{6717},[\mathrm{SII}]_{6731}}\end{array}$ \\
\hline NGC 2749 & 20B & $\mathrm{E}$ & -4.8 & {$[\mathrm{NII}]_{6548}, \mathrm{H}_{\alpha},[\mathrm{NII}]_{6583},[\mathrm{SII}]_{6717},[\mathrm{SII}]_{6731}$} \\
\hline NGC 2872 & $21 \mathrm{~A}$ & E & -4.8 & - \\
\hline NGC 2874 & 21B & SBbc & 4.4 & {$[\mathrm{NII}]_{6548}, \mathrm{H}_{\alpha},[\mathrm{NII}]_{6583},[\mathrm{SII}]_{6717},[\mathrm{SII}]_{6731}$} \\
\hline CGCG 008-034 & $26 \mathrm{~A}$ & $\mathrm{Sb}$ & 2.5 & {$[\mathrm{NII}]_{6583}$} \\
\hline IC $590 \mathrm{~N}$ & 26B & $\mathrm{E} / \mathrm{S} 0$ & -3.2 & $\mathrm{H}_{\alpha},[\mathrm{NII}]_{6583}$ \\
\hline CGCG 065-023 & $27 \mathrm{~A}$ & $\mathrm{~S} 0 / \mathrm{a}$ & -1.0 & {$[\mathrm{NII}]_{6548}, \mathrm{H}_{\alpha},[\mathrm{NII}]_{6583}$} \\
\hline UGC 5627 & 27B & Sab & 1.7 & {$[\mathrm{NII}]_{6548}, \mathrm{H}_{\alpha},[\mathrm{NII}]_{6583}$} \\
\hline NGC 4003 & $39 \mathrm{~A}$ & SB0 & -1.9 & $\mathrm{H}_{\alpha},[\mathrm{NII}]_{6583}$ \\
\hline NGC 4002 & 39B & $\mathrm{Sa}$ & 1.4 & {$[\mathrm{NIII}]_{6583}$} \\
\hline NGC 4446 & $49 \mathrm{~A}$ & $\mathrm{Sc}$ & 5.8 & $\mathrm{H}_{\beta},[\mathrm{OI}]_{6300},[\mathrm{NII}]_{6548}, \mathrm{H}_{\alpha},[\mathrm{NII}]_{6583}$ \\
\hline NGC 4447 & 49B & SB0 & -2.2 & - \\
\hline CGCG 162-059 & $61 \mathrm{~A}$ & SBbc & 4.4 & {$[\mathrm{NII}]_{6548}, \mathrm{H}_{\alpha},[\mathrm{NII}]_{6583}$} \\
\hline UGC 9012 & $61 \mathrm{~B}$ & So & -2.0 & - \\
\hline UGC 9413 & $65 \mathrm{~A}$ & Sbc & 4.0 & {$[\mathrm{OII}]_{3727}, \mathrm{H}_{\beta},[\mathrm{OIII}]_{4959},[\mathrm{OIII}]_{5007},[\mathrm{NII}]_{6548}, \mathrm{H}_{\alpha},[\mathrm{NII}]_{6583}, \mathrm{HeI}_{6678}[\mathrm{SII}]_{6717},[\mathrm{SII}]_{6731}$} \\
\hline CGCG 353-044 & 65B & S0 & -1.7 & - \\
\hline NGC 5771 & $67 \mathrm{~A}$ & E & -4.5 & - \\
\hline NGC 5773 & 67B & $\mathrm{Sa}$ & 1.4 & - \\
\hline CGCG 136-013 & $75 \mathrm{~A}$ & E? & -0.8 & {$[\mathrm{NII}]_{6583}$} \\
\hline CGCG 136-015 & 75B & S? & 2.2 & {$[\mathrm{NIII}]_{6548}, \mathrm{H}_{\alpha},[\mathrm{NII}]_{6583}$} \\
\hline NGC 6251 & $79 \mathrm{~A}$ & E & -4.8 & {$[\mathrm{OIII}]_{5007},[\mathrm{NII}]_{6548}, \mathrm{H}_{\alpha},[\mathrm{NII}]_{6583}$} \\
\hline NGC 6252 & 79B & S? & 4.4 & {$[\mathrm{NII}]_{6583}$} \\
\hline IC 5285 & $84 \mathrm{~A}$ & E? & -0.8 & {$[\mathrm{NII}]_{6583}$} \\
\hline NGC 7489 & 84B & $\mathrm{Sc}$ & 6.5 & $\mathrm{H}_{\alpha},[\mathrm{NII}]_{6583}$ \\
\hline
\end{tabular}

some UZC-BGP systems in the close $\left(200 h^{-1}\right) \mathrm{kpc}$ and/or distant $\left(1 h^{-1}\right) \mathrm{Mpc}$ surrounding area do not play a role comparable to that of luminous member galaxies.

The lower panels of Fig. 3 indicate a "dynamical" difference between pairs containing only spirals (middle panel) and pairs containing either both (left panel) or at least one (right panel) early-type galaxy, the former being characterized by a narrower $\left|\Delta v_{\mathrm{r}}\right|$ distribution than the latter ones (KS confidence level $93.9 \%$ and $99.9 \%$ respectively). This difference (already outlined in Paper I) suggests that $\mathrm{E}+\mathrm{S}$ and $\mathrm{E}+\mathrm{E}$ pairs might be embedded within large loose structures as their broader $\left|\Delta v_{\mathrm{r}}\right|$ distribution indicates the presence of a larger potential well. In this framework the emission spectrum of the 3 early-type galaxies belonging to the $\mathrm{E}+\mathrm{E}$ pairs could arise from infalling of these galaxies within a loose group as suggested from their large $\left|\Delta v_{\mathrm{r}}\right|$ value. We have checked this hypothesis and found that neither UZCBGP 51 nor UZC-BGP 77 are part of any known galaxy group. However, inspection of available (LEDA) redshifts of galaxies in the environment surrounding both pairs show that UZC-BGP 51 might be part of a loose galaxy group which possibly could be infalling on the nearby $\left(4 h^{-1} \mathrm{Mpc}\right)$ Coma cluster. UZC-BGP 77A
(NGC 6018) might be infalling on a galaxy loose group having the same radial velocity as UZC-BGP 77B (NGC 6021). More investigation is required to confirm our hypothesis.

\section{Nuclear activity classification}

To classify nuclear activity in our galaxy sample, we have made use of the standard diagnostic diagrams (Baldwin et al. 1981; Veilleux \& Osterbrock 1987; Veilleux 2002), also known as the BPT diagrams, which have proved to be an extremely efficient method to distinguish the different types of activity encountered in emission line galaxies (Veilleux et al. 1995; Véron et al. 1997; Gonçalves et al. 1999). Moreover, being based on ratios of emission lines which are very close in wavelength they are almost unaffected by reddening corrections (Veilleux \& Osterbrock 1987). The diagnostic diagrams relate $[\mathrm{OIII}]_{5007} / \mathrm{H}_{\beta}$ to $[\mathrm{NII}]_{6583} / \mathrm{H}_{\alpha}$, $[\mathrm{SII}]_{(6717+6731)} / \mathrm{H}_{\alpha}$ and $[\mathrm{OI}]_{6300} / \mathrm{H}_{\alpha}$.

Of the 68 galaxies with emission spectra (cf. Tables 1-3), 24 allowed us to create from one to three of the abovementioned standard diagnostic diagrams. These galaxies are listed in Table 4, in order of decreasing number of diagnostic diagrams, 

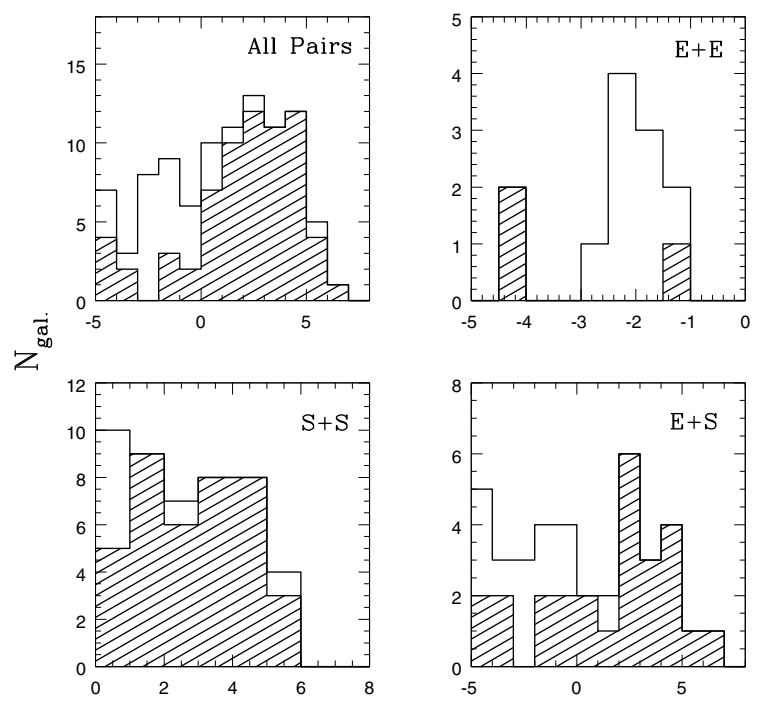

$\mathrm{T}$

Fig. 1. Relation between pair morphology and the presence of emission lines in the member galaxy spectra. The continuous histogram shows the morphological distribution of the whole galaxy pair sample (upper left), and of $\mathrm{E}+\mathrm{E}$ (upper right), $\mathrm{S}+\mathrm{S}$ (lower left) and $\mathrm{E}+\mathrm{S}$ (lower right) pairs. Dashed histograms indicate the morphological distribution of galaxies with the emission line spectrum in each sample.
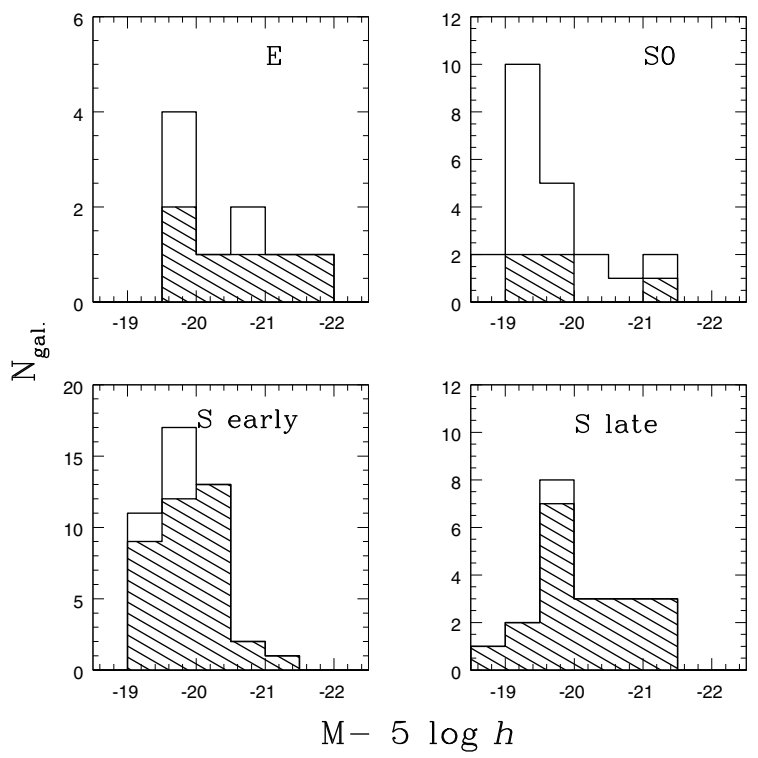

Fig. 2. Absolute magnitude $\left(M_{B}\right)$ distribution of galaxies, of different morphological types, having or not having emission lines in their spectra. Continuous distribution refer to the whole samples, hatched one to galaxies with emission lines.

i.e. the first 14 galaxies have all line ratios measured, the subsequent 8 galaxies only three line ratios and the remaining 2 only two. Table 4 reports for these galaxies, the UZC-BGP identifier (Col. 1), pair/galaxy morphology (Col. 2, galaxy morphology is underlined in cases of mixed pair morphology), line ratios and errors (Cols. 3-6). Each line ratio has been obtained by averaging line ratios obtained independently by each of us and the associated error represents the standard deviation $(\sigma)$. The vast majority (20/24) of galaxies listed in Table 4 belong to the $S+S$ pair sample and the remaining 4 are spirals, implying that there are no early-type galaxies in our sample showing at least 4 emission lines.
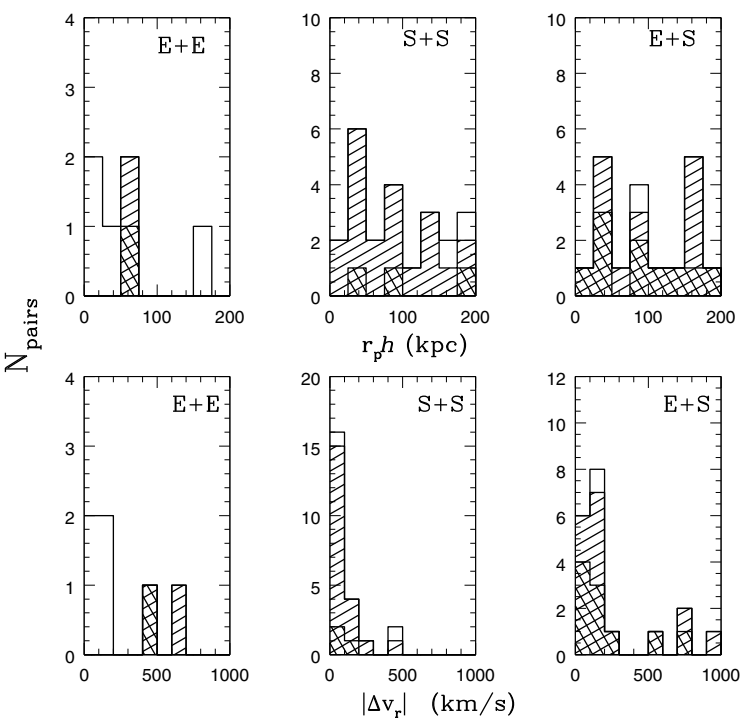

Fig. 3. Relation between the presence of emission lines in the galaxy spectra and dynamical parameters of the pairs. The upper panels show the galaxy-galaxy projected distance $\left(r_{\mathrm{p}}\right)$ distribution, the lower panels the velocity difference $\left(\left|\Delta v_{\mathrm{r}}\right|\right)$ between galaxies (in each pair) for $\mathrm{E}+\mathrm{E}$, $\mathrm{S}+\mathrm{S}, \mathrm{E}+\mathrm{S}$ respectively. Pairs having at least one galaxy with an emission spectrum are represented by the dashed histogram. The single dash indicates pairs in which both members have an emission line spectrum, the double dash pairs hosting only one emission line galaxy.

The $\log \left([\mathrm{OIII}]_{5007} / \mathrm{H}_{\beta}\right)$ versus $\log \left([\mathrm{NII}]_{6583} / \mathrm{H}_{\alpha}\right)$ diagnostic diagram (hereafter DD1), for all the galaxies listed in Table 4, is shown in Fig. 4. Different symbols indicate different nuclear activity according to Veilleux \& Osterbrock (1987) empirical classification (hereafter VO87) which separates high from low excitation galaxies (Seyfert 2 from LINERs and HII galaxies from $\mathrm{SBs}$ ) based on a $[\mathrm{OIII}]_{5007} / \mathrm{H}_{\beta}$ value of 3 and LINER from SBs based on a $[\mathrm{NII}]_{6583} / \mathrm{H}_{\alpha}$ value of 0.6.

According to VO87, our sample contains one Seyfert 2 galaxy (triangle), two LINERs (squares) and 21 SBs (circles). The Sy 2 (UZC-BGP 83A) and the LINERs (UZC-BGP 28A and 69B) are identified in Fig. 4. On the same diagram we show two curves, the solid one to the left is the Kauffman et al. (2003) sequence (hereafter Kauff03), the dotted one to the right is the Kewley et al. (2001) sequence (hereafter Kew01). Both sequences are supposed to separate SB from AGNs. Kauff03 is an empirical sequence that has been derived from a very large sample ( 22 000) of SDSS emission line galaxies, while Kew01 is a theoretical sequence derived using a wide set of models accounting for photoionization and stellar population synthesis. Figure 4 shows that there are 8 galaxies falling between the Kauff03 and Kew01 sequences. These galaxies, ordered by decreasing value of $[\mathrm{OIII}]_{5007} / \mathrm{H}_{\beta}$, are UZC-BGP $81 \mathrm{~A}, 66 \mathrm{~A}, 24 \mathrm{~A}, 5 \mathrm{~A}, 44 \mathrm{~A}, 69 \mathrm{~B}$ (a LINER according to VO87), $2 \mathrm{~A}$ and $81 \mathrm{~B}$ and are indicated in Fig. 4. Two of these galaxies (UZC-BGP 81A and 66A) may fall just below the Kauff03 sequence taking into account the error associated with the $[\mathrm{OIII}]_{5007} / \mathrm{H}_{\beta}$ measurement, while another one (UZC-BGP 81B) could move from just above the sequence to the sequence itself. One further galaxy (UZC-BGP 74A) is identified in Fig. 4 as, although it lies, in this diagram, well below the Kauff03 sequence, it occupies the LINER region of Fig. 6.

Figure 5 shows the $\log \left([\mathrm{OIII}]_{5007} / \mathrm{H}_{\beta}\right)$ versus $\log \left([\mathrm{SII}]_{(6717+6731)} / \mathrm{H}_{\alpha}\right)$ diagram (hereafter DD2) for the 22 galaxies of Table 4 which also have these line ratios measured. As in Fig. 4 different symbols stand for different classifications in the VO87 scheme $\left([\mathrm{OIII}]_{5007} / \mathrm{H}_{\beta} \geq 3\right.$ and 
Table 4. Spectral line ratios for standard diagnostic diagrams (DDs 1, 2 and 3).

\begin{tabular}{|c|c|c|c|c|c|}
\hline UZC-BGP & Pair type & {$[\mathrm{OIIII}]_{5007} / \mathrm{H}_{\beta}$} & {$[\mathrm{NIII}]_{6583} / \mathrm{H}_{\alpha}$} & {$[\mathrm{SII}]_{6717+6731) / \mathrm{H}_{\alpha}}$} & {$[\mathrm{OI}]_{6300} / \mathrm{H}_{\alpha}$} \\
\hline $2 \mathrm{~A}$ & $\mathrm{~S}+\mathrm{S}$ & $0.49 \pm 0.06$ & $0.553 \pm 0.006$ & $0.333 \pm 0.004$ & $0.043 \pm 0.002$ \\
\hline $9 \mathrm{~A}$ & $\mathrm{~S}+\mathrm{S}$ & $1.59 \pm 0.06$ & $0.198 \pm 0.003$ & $0.382 \pm 0.006$ & $0.035 \pm 0.006$ \\
\hline $14 \mathrm{~A}$ & $\mathrm{E}+S$ & $0.516 \pm 0.022$ & $0.349 \pm 0.006$ & $0.278 \pm 0.023$ & $0.022 \pm 0.008$ \\
\hline $20 \mathrm{~A}$ & $\mathrm{E}+\bar{S}$ & $1.32 \pm 0.06$ & $0.30 \pm 0.01$ & $0.36 \pm 0.02$ & $0.023 \pm 0.002$ \\
\hline $24 \mathrm{~A}$ & $\mathrm{~S}+\overline{\mathrm{S}}$ & $1.61 \pm 0.09$ & $0.55 \pm 0.02$ & $0.25 \pm 0.03$ & $0.045 \pm 0.000$ \\
\hline $28 \mathrm{~A}$ & $\mathrm{~S}+\mathrm{S}$ & $2.3 \pm 0.4$ & $1.16 \pm 0.02$ & $0.70 \pm 0.05$ & $0.40 \pm 0.04$ \\
\hline $28 \mathrm{~B}$ & $\mathrm{~S}+\mathrm{S}$ & $0.67 \pm 0.02$ & $0.276 \pm 0.002$ & $0.19 \pm 0.01$ & $0.023 \pm 0.006$ \\
\hline $29 \mathrm{~B}$ & $\mathrm{~S}+\mathrm{S}$ & $0.60 \pm 0.05$ & $0.287 \pm 0.004$ & $0.20 \pm 0.01$ & $0.021 \pm 0.003$ \\
\hline $68 \mathrm{~A}$ & $\mathrm{~S}+\mathrm{S}$ & $0.79 \pm 0.02$ & $0.25 \pm 0.01$ & $0.21 \pm 0.02$ & $0.044 \pm 0.007$ \\
\hline $74 \mathrm{~A}$ & $\mathrm{~S}+\mathrm{S}$ & $0.92 \pm 0.03$ & $0.35 \pm 0.02$ & $0.22 \pm 0.01$ & $0.54 \pm 0.03$ \\
\hline $74 \mathrm{~B}$ & $\mathrm{~S}+\mathrm{S}$ & $1.6 \pm 0.1$ & $0.18 \pm 0.02$ & $0.232 \pm 0.003$ & $0.05 \pm 0.01$ \\
\hline $81 \mathrm{~B}$ & $\mathrm{~S}+\mathrm{S}$ & $0.36 \pm 0.04$ & $0.509 \pm 0.009$ & $0.17 \pm 0.02$ & $0.028 \pm 0.003$ \\
\hline $83 \mathrm{~A}$ & $\mathrm{~S}+\mathrm{S}$ & $4.4 \pm 0.3$ & $0.332 \pm 0.003$ & $0.144 \pm 0.006$ & $0.078 \pm 0.005$ \\
\hline $83 \mathrm{~B}$ & $\mathrm{~S}+\mathrm{S}$ & $0.89 \pm 0.03$ & $0.262 \pm 0.006$ & $0.26 \pm 0.01$ & $0.044 \pm 0.003$ \\
\hline $5 \mathrm{~A}$ & $\mathrm{E}+\underline{S}$ & $1.45 \pm 0.02$ & $0.387 \pm 0.005$ & $0.248 \pm 0.005$ & - \\
\hline $22 \mathrm{~A}$ & $\mathrm{~S}+\overline{\mathrm{S}}$ & $0.79 \pm 0.02$ & $0.38 \pm 0.01$ & $0.17 \pm 0.01$ & - \\
\hline $22 \mathrm{~B}$ & $\mathrm{~S}+\mathrm{S}$ & $0.64 \pm 0.03$ & $0.33 \pm 0.01$ & $0.28 \pm 0.02$ & - \\
\hline $29 \mathrm{~A}$ & $\mathrm{~S}+\mathrm{S}$ & $0.28 \pm 0.04$ & $0.442 \pm 0.004$ & $0.102 \pm 0.008$ & - \\
\hline $66 \mathrm{~A}$ & $\mathrm{~S}+\mathrm{S}$ & $2.3 \pm 0.4$ & $0.27 \pm 0.01$ & $0.198 \pm 0.004$ & - \\
\hline $69 \mathrm{~B}$ & $\mathrm{~S}+\mathrm{S}$ & $0.79 \pm 0.02$ & $0.76 \pm 0.02$ & $0.51 \pm 0.03$ & - \\
\hline $81 \mathrm{~A}$ & $\mathrm{~S}+\mathrm{S}$ & $3.0 \pm 0.3$ & $0.203 \pm 0.004$ & $0.18 \pm 0.02$ & - \\
\hline $82 \mathrm{~A}$ & $\mathrm{~S}+\mathrm{S}$ & $0.42 \pm 0.04$ & $0.386 \pm 0.004$ & $0.18 \pm 0.02$ & - \\
\hline $44 \mathrm{~A}$ & $\mathrm{~S}+\mathrm{S}$ & $1.3 \pm 0.2$ & $0.39 \pm 0.01$ & - & - \\
\hline $65 \mathrm{~A}$ & $\mathrm{E}+S$ & $0.97 \pm 0.05$ & $0.263 \pm 0.008$ & - & - \\
\hline
\end{tabular}

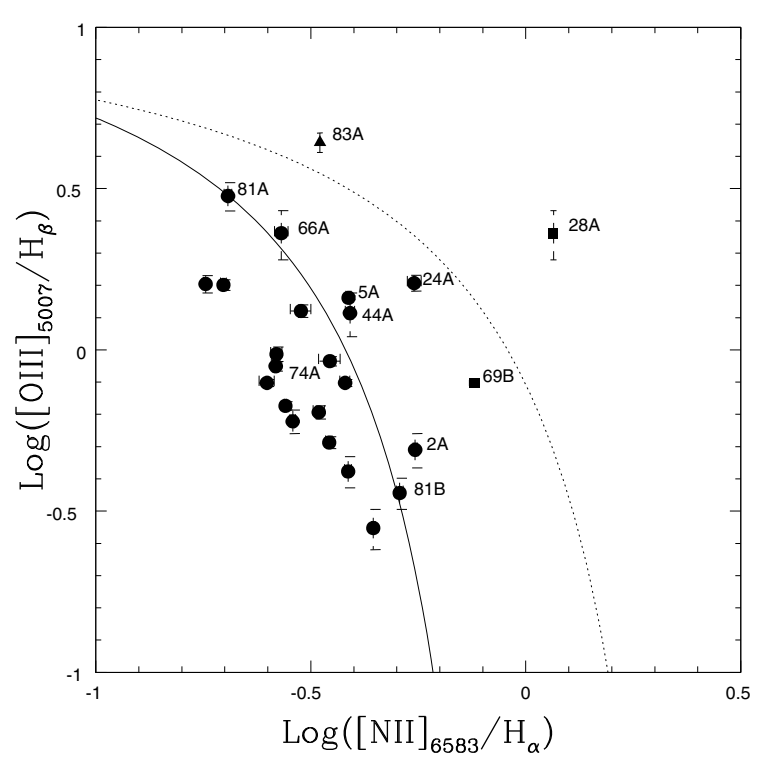

Fig. 4. $\log \left([\mathrm{OIII}]_{5007} / \mathrm{H}_{\beta}\right)$ versus $\log \left([\mathrm{NII}]_{6583} / \mathrm{H}_{\alpha}\right)$ diagnostic diagram (DD1). Circles, squares and the triangle indicate classification into SB, LINER and Sy 2 according to the VO87 scheme. The solid curve on the left is the Kauff03 sequence, while the dotted curve on the right corresponds to the Kew01 sequence. Both sequences are supposed to separate SB from AGNs. Identification is provided for all galaxies above the Kauff03 sequence as, according to it, they should all be classified as AGNs. One galaxy (UZC-BGP 74A) below the Kauff03 sequence has been identified too as it lies in the LINER region of Fig. 6.

$[\mathrm{SII}]_{(6717+6731)} / \mathrm{H}_{\alpha} \geq 0.4$ separate, respectively, Sy $2 \mathrm{~s}$ and LINERs from SBs). The VO87 criteria confirm the classification as Sy 2 and LINERs obtained from Fig. 4 for UZC 83A, 28A and 69B. The dotted curve and line correspond to Kew01 and Kew06 (Kewley et al. 2006) sequences. The former separates SB (below) from AGNs (above), the latter Sy 2 (above) from LINERs (below). We also show on this plot

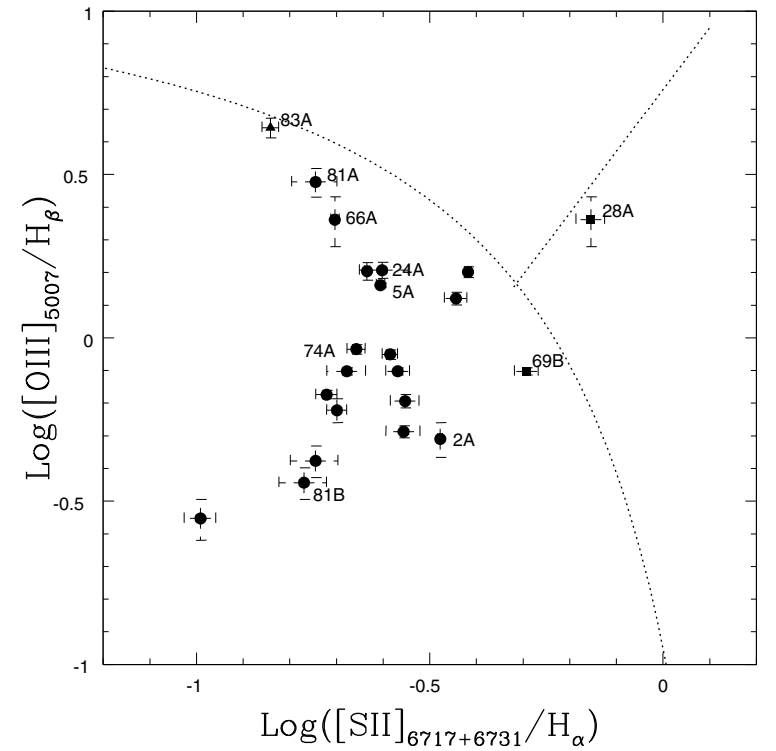

Fig. 5. $\log \left([\mathrm{OIII}]_{5007} / \mathrm{H}_{\beta}\right)$ versus $\log \left([\mathrm{SII}]_{(6717+6731)} / \mathrm{H}_{\alpha}\right)$ diagnostic diagram (DD2). In analogy with Fig. 4, circles, squares and the triangle indicate classification into SB, LINER and Sy 2 according to the VO87 scheme. The dotted curve represents the Kew01 sequence separating the SB (below) from AGN (above) region. The dotted line is the Kew06 sequence which separates Seyferts (above) from LINERs (below). Identified on this plot are all the galaxies above the Kauff03 sequence in Fig. 4 and UZC-BGP 74A.

the three confirmed (VO87) AGNs and 7 (of the 8) galaxies lying between the Kauff03 and Kew01 sequences in Fig. 4, as UZC-BGP 44A has only DD1 available (cf. Table 4). Finally the position of UZC-BGP 74A is indicated, since this galaxy occupies the LINER region of Fig. 6.

Figure 5 shows that, according to the Kew01 and Kew06 classification, only one galaxy (UZC-BGP 28A) would be classified as a LINER. All the others would be SB, although 


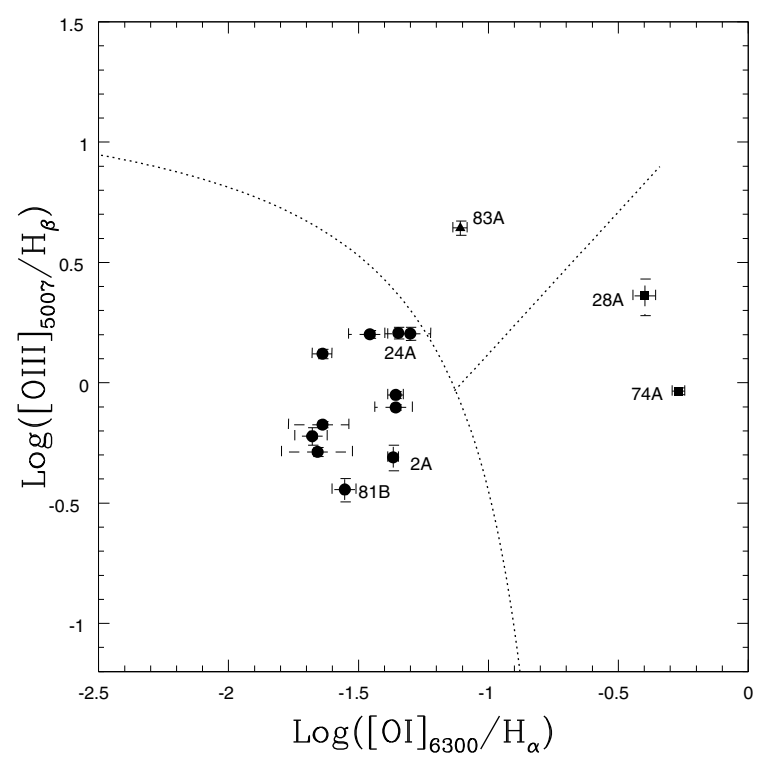

Fig. 6. $\log \left([\mathrm{OIII}]_{5007} / \mathrm{H}_{\beta}\right)$ versus $\log \left([\mathrm{OI}]_{6300} / \mathrm{H}_{\alpha}\right)$ diagnostic diagram (DD3). Symbols are as in Figs. 4 and 5. Dotted curve and line represent Kew01 and Kew06 sequences separating, respectively, SB from AGNs and Seyfert from LINERs. Identification is provided for all the galaxies lying in the "AGN" region either in this or in another diagnostic diagrams.

UZC-BGP 83A (Sy 2 following the VO87 scheme) lies just below the Kew01 sequence and would move exactly on to it if one lets $[\mathrm{OIII}]_{5007} / \mathrm{H}_{\beta}$ attain its maximum possible value (within the error).

Figure 6 shows the $\log \left([\mathrm{OIII}]_{5007} / \mathrm{H}_{\beta}\right)$ versus $\log \left([\mathrm{OI}]_{6300} / \mathrm{H}_{\alpha}\right)$ diagram (hereafter DD3) for the 14 galaxies in Table 4 with those measured line ratios. Symbols indicate different nuclear activity type according to the VO87 scheme that separates Sy 2 from SB and LINERs based on a value of $[\mathrm{OIII}]_{5007} / \mathrm{H} \beta$ of 3 and requires a value of $[\mathrm{OI}]_{6300} / \mathrm{H} \alpha \geq 0.08$ for Sy 2 and $\geq 0.17$ for LINERs. Here the dotted curve and line correspond to Kew01 and Kew06 sequences separating SB from AGNs and Sy 2 from LINERs respectively. We indicate, in this plot, the galaxies lying above the Kew01 curve and 3 (over 8) galaxies lying in the Kauff03 Kew01 region of DD1 (Fig. 4). Two galaxies (UZC-BGP 28A and 74A) occupy the Kew01-Kew06 LINER region, one (UZC-BGP 83A) the Sy 2 region. This classification is confirmed by VO87. One galaxy in Fig. 6 (UZC-BGP 74B) could move from the SB locus to the Kew01 SB/AGN separation curve when taking into account the error associated with the $[\mathrm{OI}]_{6300} / \mathrm{H}_{\alpha}$ measurement. This galaxy is very close to UZC-BGP 24A (its right), but we have not indicated it in Fig. 6, as it occupies the SB region both in DD1 and DD2 (Figs. 4 and 5).

The spectral analysis of the 24 emission line galaxies listed in Table 4 allow us to unambiguously classify 14 of them, as for these galaxies all the different classification schemes (V087, Kauff03 and Kew01-06) give consistent results on the 3 diagnostic diagrams. Of the remaining 10 galaxies 8 lie above the Kauff03 sequence and below the Kew01 in DD1. One of these 8 (UZC-BGP 44A) has only DD1 available, 4 also have DD2, and 3 have both DD2 and DD3 available. The VO87 and Kew01 classification schemes agree on SB activity in the other diagnostic diagrams for 6 of the 7 galaxies having either both DD2 and DD3 or only DD2 available; only UZC-BGP 69B is classified (in DD2) as a LINER in the VO87 scheme and as a SB in the Kew01 one. The 2 other "difficult" cases are UZC-BGP 83A
Table 5. Spectral line ratios for DD4.

\begin{tabular}{|c|c|c|c|}
\hline UZC-BGP & Pair type & {$[\mathrm{NII}]_{6583} / \mathrm{H}_{\alpha}$} & {$[\mathrm{NII}]_{6583}$} \\
\hline $3 \mathrm{~A}$ & $\mathrm{E}+\underline{S}$ & $0.45 \pm 0.01$ & $5.1 \pm 0.8$ \\
\hline $4 \mathrm{~B}$ & $\mathrm{E}+\bar{S}$ & $2.99 \pm 0.01$ & $1.01 \pm 0.01$ \\
\hline 8B & $\mathrm{S}+\overline{\mathrm{S}}$ & $0.34 \pm 0.02$ & $4.2 \pm 0.3$ \\
\hline 9B & $\mathrm{S}+\mathrm{S}$ & $0.332 \pm 0.004$ & $3.71 \pm 0.06$ \\
\hline $10 \mathrm{~A}$ & $E+S$ & $1.27 \pm 0.08$ & $2.11 \pm 0.04$ \\
\hline $10 \mathrm{~B}$ & $\overline{\mathrm{E}}+S$ & $0.85 \pm 0.02$ & $2.9 \pm 0.1$ \\
\hline 17B & $\mathrm{S}+\overline{\mathrm{S}}$ & $0.337 \pm 0.004$ & $5.97 \pm 0.04$ \\
\hline $18 \mathrm{~A}$ & $\mathrm{E}+\underline{S}$ & $0.245 \pm 0.007$ & $12.0 \pm 0.4$ \\
\hline $20 \mathrm{~B}$ & $\underline{E}+\bar{S}$ & $2.9 \pm 0.1$ & $2.9 \pm 0.1$ \\
\hline $21 \mathrm{~B}$ & $\overline{\mathrm{E}}+\underline{S}$ & $9.4 \pm 1.6$ & $2.8 \pm 0.2$ \\
\hline $24 \mathrm{~B}$ & $\mathrm{~S}+\overline{\mathrm{S}}$ & $0.61 \pm 0.02$ & $6.4 \pm 0.3$ \\
\hline $26 \mathrm{~B}$ & $E+S$ & $2.8 \pm 0.8$ & $1.3 \pm 0.3$ \\
\hline $27 \mathrm{~A}$ & $\bar{E}+S$ & $3.0 \pm 0.4$ & $4.76 \pm 0.07$ \\
\hline 27B & $\overline{\mathrm{E}}+\underline{S}$ & $0.50 \pm 0.04$ & $4.4 \pm 0.2$ \\
\hline $36 \mathrm{~A}$ & $\mathrm{~S}+\overline{\mathrm{S}}$ & $2.5 \pm 0.4$ & $3.5 \pm 0.6$ \\
\hline $36 \mathrm{~B}$ & $\mathrm{~S}+\mathrm{S}$ & $0.58 \pm 0.02$ & $5.8 \pm 0.2$ \\
\hline $37 \mathrm{~A}$ & $\mathrm{~S}+\mathrm{S}$ & $0.70 \pm 0.03$ & $3.6 \pm 0.3$ \\
\hline 37B & $\mathrm{S}+\mathrm{S}$ & $0.36 \pm 0.02$ & $5.5 \pm 0.5$ \\
\hline $39 \mathrm{~A}$ & $\underline{E}+\mathrm{S}$ & $0.838 \pm 0.006$ & $6.4 \pm 0.1$ \\
\hline 44B & $\overline{\mathrm{S}}+\mathrm{S}$ & $0.57 \pm 0.02$ & $6.2 \pm 0.2$ \\
\hline $49 \mathrm{~A}$ & $\mathrm{E}+\underline{S}$ & $0.375 \pm 0.005$ & $7.7 \pm 0.3$ \\
\hline 59B & $\mathrm{S}+\overline{\mathrm{S}}$ & $0.650 \pm 0.008$ & $6.10 \pm 0.07$ \\
\hline $61 \mathrm{~A}$ & $\mathrm{E}+\underline{S}$ & $0.752 \pm 0.07$ & $7.8 \pm 0.3$ \\
\hline $68 \mathrm{~B}$ & $\mathrm{~S}+\overline{\mathrm{S}}$ & $0.36 \pm 0.01$ & $4.0 \pm 0.3$ \\
\hline 71B & $\mathrm{S}+\mathrm{S}$ & $3.2 \pm 0.1$ & $3.8 \pm 0.2$ \\
\hline $75 \mathrm{~A}$ & $E+S$ & $1.565 \pm 0.007$ & $2.55 \pm 0.07$ \\
\hline $75 B$ & $\overline{\mathrm{E}}+\underline{S}$ & $0.95 \pm 0.01$ & $2.7 \pm 0.3$ \\
\hline $77 \mathrm{~A}$ & $E+\bar{E}$ & $0.74 \pm 0.03$ & $4.5 \pm 0.3$ \\
\hline $79 \mathrm{~A}$ & $\underline{E}+\mathrm{S}$ & $1.65 \pm 0.06$ & $4.4 \pm 0.4$ \\
\hline $80 \mathrm{~B}$ & $\mathrm{~S}+\mathrm{S}$ & $0.28 \pm 0.06$ & $8.1 \pm 0.3$ \\
\hline 84B & $\mathrm{E}+S$ & $0.44 \pm 0.02$ & $4.42 \pm 0.02$ \\
\hline
\end{tabular}

and 74A. The first one is a Sy 2 in both DD1 and DD3, for all classification schemes, but in DD2 is a SB, according to Kew01 (although it lies very close to the SB/AGN border and would lie exactly on it if $[\mathrm{OIII}]_{5007} / \mathrm{H} \beta$ attains its maximum permitted value within the error), and a Sy 2 according to VO87. More "difficult" is the case of UZC-BGP 74A which is a LINER in DD3 (for both Kew01 and VO87) and a SB in DD1 and DD2 for all classification schemes.

Galaxies displaying different kinds of nuclear activity in different diagnostic diagrams should be classified as composite objects (Kewley et al. 2006). However, in order to avoid a double classification (e.g. SB/AGN, Sy2/SB, LINER/SB) we have assigned to each galaxy the "most frequent" classification according to the different schemes applied to the 3 diagnostic diagrams. However, we record each classification scheme in each diagnostic diagram in Table 6, which summarizes the results of our spectral analysis.

A large fraction of the emission line galaxies (31/68) have well defined $\mathrm{H}_{\alpha}$ and [NII] lines but do not show [OIII] and/or $\mathrm{H}_{\beta}$ features and thus does not allow us to use the standard diagnostic diagrams. For these galaxies, listed in Table 5, the activity type can be classified (Coziol et al. 1998) comparing the $E W$ of the $[\mathrm{NII}]_{6583}$ feature with the ratio of $[\mathrm{NII}]_{6583}$ to $\mathrm{H}_{\alpha}$. These values are listed in Table 5, together with their errors, which, similarly to Table 4, represent the average of 3 measures obtained independently by each of us and the associated $\sigma$. The underlined morphology in Col. 2 indicates, as in Table 4, the "rough" galaxy morphology when the galaxy pair is of mixed $(E+S)$ type.

Following Coziol et al. (1998) we have classified the 31 galaxies listed in Table 5 as SB if they have $\log \left([\mathrm{NII}]_{6583} / \mathrm{H}_{\alpha}\right)<-0.4$ and $\log E W\left([\mathrm{NII}]_{6583}\right) \geq 0.5 ; \mathrm{AGN}$ 


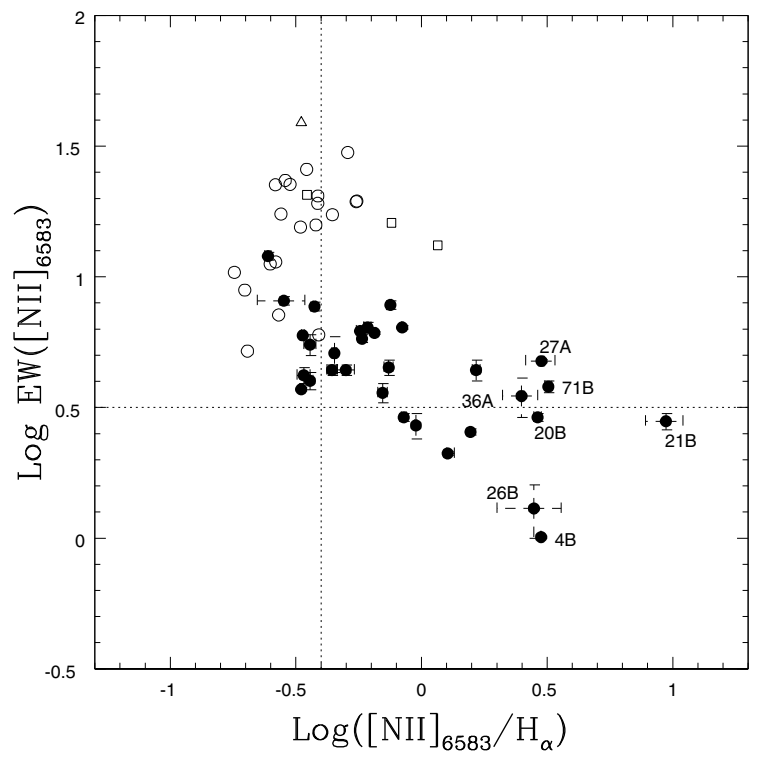

Fig. 7. $\log E W\left([\mathrm{NII}]_{6583}\right)$ versus $\log \left([\mathrm{NII}]_{6583} / \mathrm{H}_{\alpha}\right)$ diagnostic diagram (DD4) that we have used to classify the 31 galaxies listed in Table 5 (filled circles). The vertical dotted line separates the SB locus from the AGN one, the horizontal dotted line separates AGNs (up) from LLAGNs (low). The open symbols indicate the position in this diagram of the 24 galaxies listed in Table 4 , whose nuclear activity has been classified by means of one or more standard diagnostic diagrams. (Circles, squares and triangle indicate classification into SB, LINER and Sy 2 according to the V087 scheme applied to DDs 1, 2 and 3.)

and LLAGN if they have $\log E W\left([\mathrm{NII}]_{6583} / \mathrm{H}_{\alpha}\right) \geq-0.4$ and $\log E W\left([\mathrm{NII}]_{6583}\right) \geq 0.5$ or $<0.5$. The lines which separate the loci of SB, AGN and LLAGN are represented in Fig. 7 which shows the $\log E W\left([\mathrm{NII}]_{6583}\right)$ versus $\log \left([\mathrm{NII}]_{6583} / \mathrm{H}_{\alpha}\right)$ diagram (hereafter DD4) for the 31 galaxies listed in Table 5 (filled circles). Among these galaxies we have identified the seven showing the largest value of $[\mathrm{NII}]_{6583} / \mathrm{H}_{\alpha}$. This behaviour is due to the presence of a strong $\mathrm{H}_{\alpha}$ feature in absorption depressing the emission feature and resulting in an enhanced $[\mathrm{NII}]_{6583} / \mathrm{H}_{\alpha}$ ratio. From Fig. 7 we see that only one galaxy (UZC-BGP 36A) could have its classification changed (from AGN to LLAGN) when taking into account the error associated with $E W\left([\mathrm{NII}]_{6583}\right)$

The classification scheme adopted by Coziol et al. (1998) is empirical and might, thus, be questioned. For this reason we also show, on the same plot (Fig. 7), the 24 galaxies (open symbols) listed in Table 4 that we have classified with one or more standard diagnostic diagrams. The different symbols indicate (as in Figs. 4-6) different nuclear activity. From Fig. 7 we see that for low values of $E W\left([\mathrm{NII}]_{6583}\right)\left(\log E W\left([\mathrm{NII}]_{6583} \sim<1.1\right)\right.$ the Coziol classification scheme holds, since all galaxies classified as SB (open circles) occupy the SB region of the diagram, whereas for larger values of $E W\left([\mathrm{NII}]_{6583}\right)$ there are SBs in the AGN region and AGN in the SB ones ${ }^{2}$. The agreement between Coziol and the standard classification, occurring at low values of $E W\left([\mathrm{NII}]_{6583}\right)$ where SBs from Tables 4 and 5 overlap, increases our confidence in this classification scheme.

Of the remaining 13 galaxies with emission lines (cf. Tables $1-3), 10$ show only the $[\mathrm{NII}]_{6583}$ emission feature and thus, according to Coziol et al. (1998), we have classified them

2 This behaviour is not unexpected since the sequence that separates AGN from SBs, in Fig. 4, is bended in $[\mathrm{NII}]_{6583} / \mathrm{H}_{\alpha}$ and Sy 2 are characterized by lower values of $[\mathrm{NII}]_{6583} / \mathrm{H}_{\alpha}$ than LINERs. as LLAGN candidates, while 3 show only $\mathrm{H}_{\alpha}$ in emission and have not been classified.

Table 6 summarizes the nuclear activity classification for $65 / 68$ emission line galaxies of our sample. The classification has been performed on the basis of one or more diagnostic diagrams for 55 galaxies and on the presence of the unique emission feature $[\mathrm{NII}]_{6583}$, for 10 of them, that following Coziol et al. (1998) we have classified as LLAGN candidates. Three galaxies (UZC-BGP 17A, 80A and 82B) show only $\mathrm{H}_{\alpha}$ in emission and thus could not be classified and included in Table 6. As well as the UZC-BGP identifier (Col. 1) and pair/galaxy morphology (Col. 2, as in Tables 4 and 5, galaxy morphology is underlined in $\mathrm{E}+\mathrm{S}$ pairs), Table 6 lists the classification derived from DD1, DD2 and DD3 (Cols. 3-5), according to VO87 (first subcolumn), Kew01 (second subcolumn) and Kauff03 (third subcolumn, only for DD1). In Col. 6 the classification into AGN, SB or LLAGN, according to DD4, is given. Column 7 reports the classification that we have adopted for each galaxy (LL stands for LLAGN candidate, based on the unique presence of the $[\mathrm{NII}]_{6583}$ feature). Finally, Col. 8 reports the activity type classification available for 11 galaxies from NED.

Comparison of Col. 7 with Col. 8 shows a very good agreement between our classification and the available one. The agreement is very good also for the cases in which we have adopted the "majority" classification criterion (UZC-BGP 2A, 5A and $83 \mathrm{~A})$ and when the classification has been based only on $\mathrm{H}_{\alpha}$ and $[\mathrm{NII}]_{6583}$ (UZC-BGP 18A and 37A). Only for UZC-BGP 79A (NGC 6251) could we not confirm the Sy 2 nature as we did not detect $\mathrm{H}_{\beta}{ }^{3}$ (cf. Table 3) at our imposed threshold limit for emission lines $(S / N \geq 5)$.

From Table 6 we see that among the 65 galaxies with classified nuclear activity, 29 are SB, 18 AGN (including 2 LINERs and 1 Sy 2), 8 LLAGN and 10 LLAGN candidates. SB is the most common kind of nuclear activity encountered in our sample (30\% of galaxies display it), while AGN is limited to a smaller fraction (19\%) of galaxies. SBs are more commonly found in $\mathrm{S}+\mathrm{S}$ pairs than AGNs. There are 23/29 SB galaxies and 10/18 AGNs in $\mathrm{S}+\mathrm{S}$ pairs (these last 10 include the 2 LINERs and the Sy 2), which implies a fraction of SB and AGN per spiral galaxy in $\mathrm{S}+\mathrm{S}$ pairs of $50 \%$ and $22 \%$ respectively. The remaining $6 \mathrm{SBs}$ are all hosted in the spiral member of $\mathrm{E}+\mathrm{S}$ pairs, while the 8 AGNs are equally divided between spirals and earlytype galaxies, one (UZC-BGP 77A) of these last being hosted in an E+E pair. As a whole the fractions of SB and AGNs per spiral galaxy are $45 \%$ and $22 \%$ respectively. LLAGNs, instead, are exclusively found in $\mathrm{E}+\mathrm{S}$ pairs and in half (4/8) of the cases this kind of activity is displayed by the early-type galaxy member of the pair. The distribution of LLAGN candidates (LL in Col. 7 of Table 6) appears somewhat different from the LLAGN one; there are $3 / 10 \mathrm{LL}$ in $\mathrm{S}+\mathrm{S}$ pairs and of the remaining $7 \mathrm{LL}$ only 3 are early-type galaxies. It is difficult to state whether the difference between LLAGNs and LLAGN candidates confirms previous findings (Ho et al. 1994) concerning the heterogenous nature of LLAGNs, as our classification of LLAGN candidates has been based on the presence of the $[\mathrm{NII}]_{6583}$ feature alone.

\footnotetext{
${ }^{3}$ An extremely faint $\mathrm{H}_{\beta}$ emission, largely affected by an underlying strong absorption, is present in the spectrum of this galaxy, giving a $[\mathrm{OIII}]_{5007} / \mathrm{H}_{\beta}$ value of $3.20( \pm 0.40)$. This, coupled to the $[\mathrm{NII}]_{6583} / \mathrm{H}_{\alpha}$ value of Table 5, would locate UZC-BGP 79A in the (VO87) Sy 2 region of DD1. However, since the $\mathrm{H}_{\beta}$ feature is largely below our adopted threshold we maintain for this galaxy the AGN classification that we have derived on the basis of the $[\mathrm{NII}]_{6583}$ and $\mathrm{H}_{\alpha}$ features only (DD4).
} 
Table 6. Nuclear activity classification.

\begin{tabular}{|c|c|c|c|c|c|c|c|c|c|c|c|}
\hline \multirow[t]{2}{*}{ UZC-BGP } & \multirow[t]{2}{*}{ Pair type } & \multicolumn{3}{|c|}{ DD1 } & \multicolumn{2}{|c|}{$\overline{\text { DD2 }}$} & \multicolumn{2}{|c|}{ DD3 } & \multirow[t]{2}{*}{$\overline{\text { DD4 }}$} & \multirow[t]{2}{*}{ Adopted } & \multirow[t]{2}{*}{ Known } \\
\hline & & V087 & Kew01 & Kauff03 & VO87 & Kew01 & VO87 & Kew01 & & & \\
\hline $2 \mathrm{~A}$ & $\mathrm{~S}+\mathrm{S}$ & SB & SB & $\overline{A G N}$ & SB & SB & SB & SB & & SB & SB \\
\hline $2 \mathrm{~B}$ & $\mathrm{~S}+\mathrm{S}$ & - & - & - & - & - & - & - & & LL & \\
\hline $3 \mathrm{~A}$ & $\mathrm{E}+S$ & - & - & - & - & - & - & - & AGN & AGN & LINER \\
\hline $4 \mathrm{~B}$ & $\mathrm{E}+\bar{S}$ & - & - & - & - & - & - & - & LLAGN & LLAGN & \\
\hline $5 \mathrm{~A}$ & $\mathrm{E}+\bar{S}$ & SB & SB & AGN & SB & SB & - & - & & SB & HII \\
\hline $8 \mathrm{~B}$ & $\mathrm{~S}+\overline{\mathrm{S}}$ & - & - & - & - & - & - & - & SB & SB & \\
\hline 9A & $\mathrm{S}+\mathrm{S}$ & SB & SB & SB & SB & SB & SB & SB & & SB & \\
\hline $9 \mathrm{~B}$ & $\mathrm{~S}+\mathrm{S}$ & - & - & - & - & - & - & - & SB & SB & \\
\hline $10 \mathrm{~A}$ & $E+S$ & - & - & - & - & - & - & - & LLAGN & LLAGN & \\
\hline $10 \mathrm{~B}$ & $\overline{\mathrm{E}}+S$ & - & - & - & - & - & - & - & LLAGN & LLAGN & \\
\hline $14 \mathrm{~A}$ & $\mathrm{E}+\overline{\bar{S}}$ & SB & SB & SB & SB & SB & SB & SB & & $\mathrm{SB}$ & SB \\
\hline $16 \mathrm{~B}$ & $\mathrm{E}+\bar{S}$ & - & - & - & - & - & - & - & & LL & \\
\hline 17B & $\mathrm{S}+\overline{\mathrm{S}}$ & - & - & - & - & - & - & - & SB & SB & \\
\hline $18 \mathrm{~A}$ & $\mathrm{E}+S$ & - & - & - & - & - & - & - & SB & SB & LLIRG \\
\hline $20 \mathrm{~A}$ & $\mathrm{E}+\bar{S}$ & SB & SB & SB & SB & SB & SB & SB & & SB & \\
\hline 20B & $\underline{E}+\overline{\mathrm{S}}$ & - & - & - & - & - & - & - & LLAGN & LLAGN & \\
\hline $21 \mathrm{~B}$ & $\overline{\mathrm{E}}+S$ & - & - & - & - & - & - & - & LLAGN & LLAGN & \\
\hline $22 \mathrm{~A}$ & $\mathrm{~S}+\overline{\mathrm{S}}$ & SB & SB & SB & SB & SB & - & - & & SB & \\
\hline $22 \mathrm{~B}$ & $\mathrm{~S}+\mathrm{S}$ & SB & SB & SB & SB & SB & - & - & & SB & \\
\hline $24 \mathrm{~A}$ & $\mathrm{~S}+\mathrm{S}$ & SB & SB & AGN & SB & SB & SB & SB & & SB & \\
\hline $24 \mathrm{~B}$ & $\mathrm{~S}+\mathrm{S}$ & - & - & - & - & - & - & - & AGN & AGN & \\
\hline $26 \mathrm{~A}$ & $\mathrm{E}+S$ & - & - & - & - & - & - & - & & LL & \\
\hline $26 \mathrm{~B}$ & $E+\overline{\mathrm{S}}$ & - & - & - & - & - & - & - & LLAGN & LLAGN & \\
\hline $27 \mathrm{~A}$ & $\bar{E}+\mathrm{S}$ & - & - & - & - & - & - & - & AGN & AGN & \\
\hline 27B & $\overline{\mathrm{E}}+S$ & - & - & - & - & - & - & - & AGN & AGN & \\
\hline $28 \mathrm{~A}$ & $\mathrm{~S}+\overline{\mathrm{S}}$ & LINER & LINER & AGN & LINER & LINER & LINER & LINER & & LINER & LINER \\
\hline $28 \mathrm{~B}$ & $\mathrm{~S}+\mathrm{S}$ & SB & SB & SB & SB & SB & SB & SB & & SB & \\
\hline $29 \mathrm{~A}$ & $\mathrm{~S}+\mathrm{S}$ & SB & SB & SB & SB & SB & - & - & & SB & \\
\hline $29 \mathrm{~B}$ & $\mathrm{~S}+\mathrm{S}$ & SB & SB & SB & SB & SB & SB & SB & & SB & \\
\hline $36 \mathrm{~A}$ & $\mathrm{~S}+\mathrm{S}$ & - & - & - & - & - & - & - & AGN & AGN & \\
\hline $36 \mathrm{~B}$ & $\mathrm{~S}+\mathrm{S}$ & - & - & - & - & - & - & - & AGN & AGN & \\
\hline $37 \mathrm{~A}$ & $\mathrm{~S}+\mathrm{S}$ & - & - & - & - & - & - & - & AGN & AGN & NLAGN \\
\hline $37 \mathrm{~B}$ & $\mathrm{~S}+\mathrm{S}$ & - & - & - & - & - & - & - & SB & SB & \\
\hline 39A & $E+S$ & - & - & - & - & - & - & - & AGN & AGN & \\
\hline $39 \mathrm{~B}$ & $\overline{\mathrm{E}}+S$ & - & - & - & - & - & - & - & & LL & \\
\hline $44 \mathrm{~A}$ & $\mathrm{~S}+\overline{\mathrm{S}}$ & SB & SB & AGN & - & - & - & - & & SB & \\
\hline $44 \mathrm{~B}$ & $\mathrm{~S}+\mathrm{S}$ & - & - & - & - & - & - & - & AGN & AGN & \\
\hline $49 \mathrm{~A}$ & $\mathrm{E}+\underline{S}$ & - & - & - & - & - & - & - & SB & SB & \\
\hline $51 \mathrm{~B}$ & $\mathrm{E}+\overline{\mathrm{E}}$ & - & - & - & - & - & - & - & & LL & \\
\hline $59 \mathrm{~A}$ & $\mathrm{~S}+\mathrm{S}$ & - & - & - & - & - & - & - & & LL & \\
\hline $59 \mathrm{~B}$ & $\mathrm{~S}+\mathrm{S}$ & - & - & - & - & - & - & - & AGN & AGN & \\
\hline $61 \mathrm{~A}$ & $\mathrm{E}+S$ & - & - & - & - & - & - & - & AGN & AGN & \\
\hline $65 \mathrm{~A}$ & $\mathrm{E}+\bar{S}$ & SB & SB & SB & - & - & - & - & & SB & \\
\hline $66 \mathrm{~A}$ & $\mathrm{~S}+\overline{\mathrm{S}}$ & SB & SB & AGN & SB & SB & - & - & & SB & \\
\hline $68 \mathrm{~A}$ & $\mathrm{~S}+\mathrm{S}$ & SB & SB & SB & SB & SB & SB & SB & & SB & \\
\hline $68 \mathrm{~B}$ & $\mathrm{~S}+\mathrm{S}$ & - & - & - & - & - & - & - & SB & SB & \\
\hline 69B & $\mathrm{S}+\mathrm{S}$ & LINER & SB & AGN & LINER & SB & - & - & & LINER & LLIRG \\
\hline $71 \mathrm{~A}$ & $\mathrm{~S}+\mathrm{S}$ & - & - & - & - & - & - & - & & LL & \\
\hline $71 \mathrm{~B}$ & $\mathrm{~S}+\mathrm{S}$ & - & - & - & - & - & - & - & AGN & AGN & \\
\hline $74 \mathrm{~A}$ & $\mathrm{~S}+\mathrm{S}$ & SB & SB & SB & SB & SB & LINER & LINER & & SB & \\
\hline $74 \mathrm{~B}$ & $\mathrm{~S}+\mathrm{S}$ & SB & SB & SB & SB & SB & SB & SB & & SB & \\
\hline $75 \mathrm{~A}$ & $\underline{E}+\mathrm{S}$ & - & - & - & - & - & - & - & LLAGN & LLAGN & \\
\hline $75 B$ & $\overline{\mathrm{E}}+\underline{S}$ & - & - & - & - & - & - & - & LLAGN & LLAGN & \\
\hline $77 \mathrm{~A}$ & $E+\bar{E}$ & - & - & - & - & - & - & - & AGN & AGN & \\
\hline 77B & $E+E$ & - & - & - & - & - & - & - & & LL & \\
\hline $79 \mathrm{~A}$ & $E+\mathrm{S}$ & - & - & - & - & - & - & - & AGN & AGN & Sy 2 \\
\hline $79 \mathrm{~B}$ & $\overline{\mathrm{E}}+S$ & - & - & - & - & - & - & - & & LL & \\
\hline $80 \mathrm{~B}$ & $\mathrm{~S}+\overline{\mathrm{S}}$ & - & - & - & - & - & - & - & SB & SB & \\
\hline $81 \mathrm{~A}$ & $\mathrm{~S}+\mathrm{S}$ & SB & SB & $\mathrm{SB} / \mathrm{AGN}$ & SB & SB & - & - & & SB & \\
\hline $81 \mathrm{~B}$ & $\mathrm{~S}+\mathrm{S}$ & SB & SB & AGN & SB & SB & SB & SB & & SB & \\
\hline $82 \mathrm{~A}$ & $\mathrm{~S}+\mathrm{S}$ & SB & SB & SB & SB & SB & - & - & & SB & \\
\hline $83 \mathrm{~A}$ & $\mathrm{~S}+\mathrm{S}$ & Sy 2 & AGN & AGN & Sy 2 & SB & Sy 2 & Sy 2 & & Sy 2 & Sy 2 \\
\hline $83 \mathrm{~B}$ & $\mathrm{~S}+\mathrm{S}$ & SB & SB & SB & SB & SB & SB & SB & & SB & \\
\hline $84 \mathrm{~A}$ & $\underline{E}+\mathrm{S}$ & - & - & - & - & - & - & - & & LL & \\
\hline $84 \mathrm{~B}$ & $\overline{\mathrm{E}}+S$ & - & - & - & - & - & - & - & AGN & AGN & \\
\hline
\end{tabular}



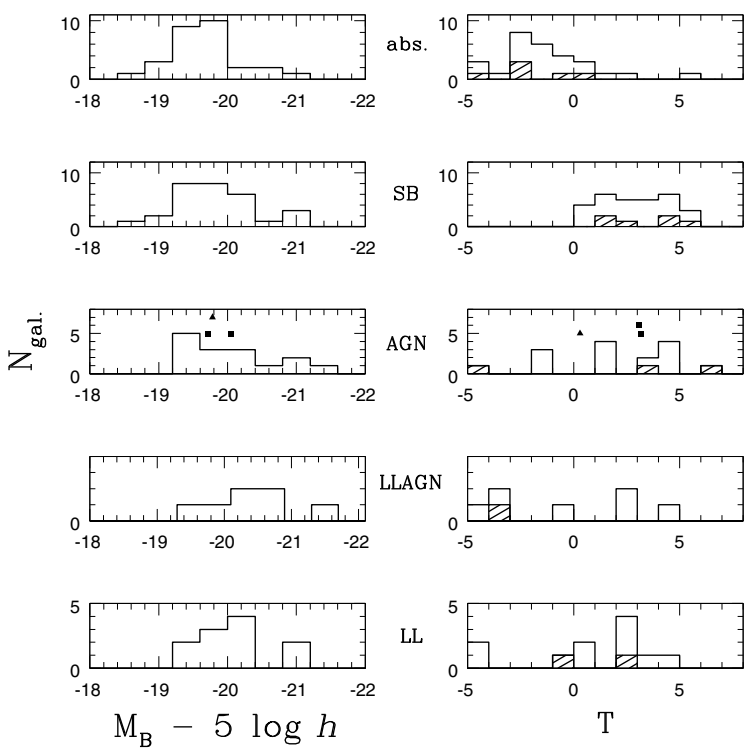

Fig. 8. Luminosity $\left(M_{B}\right)$ and morphology $(T)$ distribution of absorption line galaxies, SBs, AGNs, LLAGNs and LLAGN candidates. The dashed distributions in the right panels indicate morphological distribution of the brightest $20 \%$ galaxies in each sample. In the AGN panels the positions are indicated of the 2 LINERs (squares) and the Sy 2 (triangle) which have not been included in the histograms.

\section{The relation between nuclear activity and host galaxy}

Figure 8 shows the luminosity $\left(M_{B}\right)$ and morphology $(T)$ distribution of absorption line galaxies, SBs, AGNs, LLAGNs and LLAGN candidates. $M_{B}$ has been derived from $B_{T}$ which is available in LEDA for all but one LLAGN galaxy (UZC-BGP 10B). The dashed histogram in the morphological distribution (right panels) refers to the $20 \%$ brightest galaxies in each sample. In AGN panels magnitudes and types are also indicated of the two LINERs (squares) and the Sy 2 (triangle) which have not been included in the histograms. Examining the left panels of Fig. 8 we see that galaxies displaying the largest $B$ luminosity are LLAGNs, most $(71 \%)$ of them have $M_{B}<-20+5 \log h$, a fraction to be compared with $55 \%$ of LLAGN candidates, $44 \%$ of AGNs (LINERs and Sy 2 included), 35\% of SBs and $18 \%$ of absorption line galaxies. These last ones are of particularly low $B$ luminosity, quite unsual for passive galaxies which are, in general, much more luminous star forming ones (Kelm et al. 2005). Curiously, in our sample, absorption line galaxies and SBs have a rather similar luminosity distribution which derives from an almost "opposite" morphological content. Further investigation is needed to understand if and how this population of low luminous early-type passive galaxies is connected to the pair environment or if it is a more general characteristics of low density large scale environments.

The right panels of Fig. 8 show that absorption line galaxies are dominated by S0s $(-3 \leq T<0)$ and that very few of these galaxies display nuclear activity; ellipticals $(T<-3)$, instead, show a much higher rate of nuclear activity and in some cases (AGNs and LLAGNs) are even among the $20 \%$ brightest galaxies in each sample. Thus, low $B$ luminosity coupled with the absence of emission features seems to characterize more S0s than ellipticals, which, however, are much rarer than S0s in our sample.

The morphological distribution of SBs is rather flat as is the distribution of the brightest 6 among them. The first luminosity

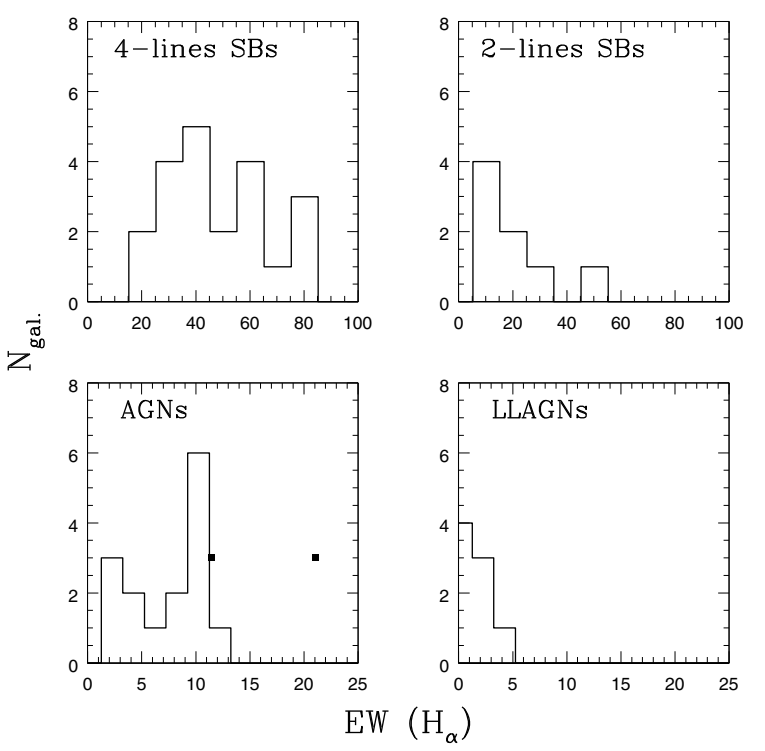

Fig. 9. $\mathrm{H}_{\alpha} E W$ distribution of SBs classified on the basis of 4 (or more) emission lines (upper left) and of 2 emission lines (upper right), AGNs (lower left) and LLAGNs (lower right). The squares in the AGN panel indicate the position that would be occupied by the two LINERs (not included in the histogram). The Sy 2 position is not indicated as its $\mathrm{H}_{\alpha}$ $E W$ is the largest in the sample (117.43 $\AA$ ) and would fall well beyond the plot limits.

ranked $\left(M_{B}=-21.15+5 \log h\right) \mathrm{SB}$ is a late spiral (UZC-BGP 9B) and the second one $\left(M_{B}=-20.82+5 \log h\right)$ UZC-BGP 2A, is an early spiral. The median type of the SB morphological distribution is 2.95 (corresponding to $\mathrm{Sb}$ ) while Ho et al. (1997) find a median value of 5 (corresponding to Sc) for their sample of galaxies with nuclear SB activity. Our sample is surely early spiral dominated but we could arrive at a larger median value of $T$ if we had more late spirals among the SBs. In our sample the fractions of early and late spiral SBs are similar (19/44 and 9/20), while Ho et al. (1997) report fractions of $38 \%$ and $82 \%$. However, if we separate 4-line SBs (i.e. SB classified on the basis of at least 4 emission lines) from 2-line SBs (classified based on $\mathrm{H}_{\alpha}$ and $[\mathrm{NII}]_{6583}$ features) we find both a $B$ luminosity increase $\left(63 \%\right.$ of these galaxies has $\left.M_{B}<-20+5 \log h\right)$ and a more advanced morphological type (median $T=3.95$ ).

The morphological distribution of AGNs is rather advanced, the median value is $2.55(\sim \mathrm{Sab})$ compared with $1(\sim \mathrm{Sa})$ of Ho et al. (1997). AGNs are spread all over the morphological range and the 3 brightest AGNs reflect this behaviour too. The brightest $\left(M_{B}=-21.6+5 \log h\right)$ AGN is UZC-BGP 79A, an elliptical galaxy (NGC 6251) classified as Sy 2 (NED) showing, in our spectrum, $\mathrm{H}_{\beta}$ emission well below our adopted $S / N$ threshold (see also Sect. 3). The other two brightest galaxies both have $M_{B}<-20.7+5 \log h$ and advanced morphological type. The LINERs and the Sy 2, whose positions are represented with two squares and a triangle, display a modest luminosity. The morphological type of the LINERs is quite advanced too; for comparison Ho et al. (1997) give a median value of 1 .

LLAGNs and LLAGN candidates are distributed over the whole morphological range, but their $T$ morphological and luminosity distributions are somewhat different, LLAGN candidates displaying more faint galaxies and spirals.

The $E W$ of the $\mathrm{H}_{\alpha}$ emission line of galaxies classified as SB (upper panels), AGN (lower left) and LLAGNs (lower right) is shown in Fig. 9. The upper left and right panels refer respectively to 4-line SBs and 2-line SBs. The squares in the AGN 
panel indicate the position that would be occupied by the two LINERs, not included in the histogram. The position of the Sy 2 is not marked as it would far to the right in this plot. This galaxy has the largest (117.43 $\AA$ ) $\mathrm{H}_{\alpha} E W$ of the whole sample.

The median values of the distribution of $\mathrm{H}_{\alpha} E W$ of 4-line SBs, 2-line SBs, AGNs and LLAGNs are respectively $45.17 \AA$, 16.48 $\AA$, $8.77 \AA$ (9.67 $\AA$ including the Sy 2 and the LINERs) and $1.33 \AA$. For comparison Miller et al. (2003) report, for a large sample of SDSS galaxies having a luminosity similar to ours but belonging to any environment, median values of the $\mathrm{H}_{\alpha} E W$ of $26 \AA, 14 \AA$ and $3 \AA$ for 4-line SBs, 2-line SBs and AGNs respectively. Thus, while the 2-line SBs display similar values of the median $\mathrm{H}_{\alpha} E W$, both 4-line $\mathrm{SBs}$ and 2-line AGNs show larger values in ours than in the Miller et al. (2003) sample. However, the criterion adopted by Miller et al. (2003) to classify 2-line AGNs is more conservative than the one adopted by us, as they required $\log \left([\mathrm{NII}] / \mathrm{H}_{\alpha}\right)>-0.2$. Application of their criterion to our data causes exclusion of 62 -line AGNs (belonging, obviously, to the large value tail of $\mathrm{H}_{\alpha} E W$ ) and reduces the median value of 2-line AGNs, in our sample, to 4.94 a value which is similar to but still exceeds (1.6 times) the one reported by Miller et al. (2003). The excess, however, disappears if we include LLAGNs in our AGN sample, as the median value of $\mathrm{H}_{\alpha}$ $E W$ drops to $2.34 \AA$, which is below the value $(3 \AA)$ reported by Miller et al. (2003). The inclusion of LLAGNs among AGNs is justified since Miller et al. (2003) state that a significant population of their 2-line AGNs are LLAGNs, although they do not say how many LLAGNs enter their AGN sample. Since LLAGNs are characterized by lower values of $\mathrm{H}_{\alpha} E W$ than AGNs, the $\mathrm{H}_{\alpha}$ $E W$ of an AGN sample also including LLAGNs will reflect the relative amount of the two populations entering the sample. For this reason we do not expect exact coincidence between the value that we derive in our sample and the one reported by Miller et al. (2003) and we consider the agreement between the two values to be quite good. Thus only 4-line SB in our sample display a median $\mathrm{H}_{\alpha} E W$ that is significantly larger than the one reported by Miller et al. (2003) and which cannot be attributed to instrumental effects as we find no correlation between the measured $\mathrm{H}_{\alpha} E W$ and the $S / N$ of the spectra.

In star forming galaxies the increase of $\mathrm{H}_{\alpha} E W$ relates to the star formation rate (SFR, Kennicutt \& Kent 1983; Kennicutt et al. 1987). Thus the larger values displayed by 4-line SB galaxies in our sample compared to the ones of Miller et al. (2003) supports the interaction - SB scenario. In this framework Barton et al. (2000) found a significant increase of $\mathrm{H}_{\alpha} E W$ with decreasing galaxy-galaxy projected distance for SB galaxies in a magnitude limited sample of galaxy pairs. The correlation holds over a limited projected distance range (from $5 h^{-1}$ to $40 h^{-1} \mathrm{kpc}$ ) over which the $\mathrm{H}_{\alpha} E W$ decreases from about $150 \AA$ to $50 \AA$. Figure 9 shows that there are no SBs in our sample with an $\mathrm{H}_{\alpha}$ $E W$ larger than $85 \AA$; that value is reached only by one galaxy (UZC-BGP 83B) whose projected distance from its companion (the only Sy 2 that we detected in our sample) is $19 h^{-1} \mathrm{kpc}$, which is small but not the smallest in the sample. The two SBs showing the smallest projected distance $\left(3 h^{-1} \mathrm{kpc}\right)$ in our sample belong to the same pair (UZC-BGP 74) and show an $\mathrm{H}_{\alpha} E W$ of 58.94 and $57.30 \AA$ respectively. Half $(15 / 29)$ of the SBs in our sample display a value of $\mathrm{H}_{\alpha} E W>40 \AA$, which is supposed to separate normal from intense SB activity (Kennicutt \& Kent 1983). None of these intense-SB galaxies is found in pairs with a projected galaxy separation $\left(r_{\mathrm{p}}\right)$ larger than $160 \mathrm{~h}^{-1}$ and 3 of them show $r_{\mathrm{p}}<30 \mathrm{~h}^{-1}$. There are no other SBs with $r_{\mathrm{p}}<30 \mathrm{~h}^{-1}$,
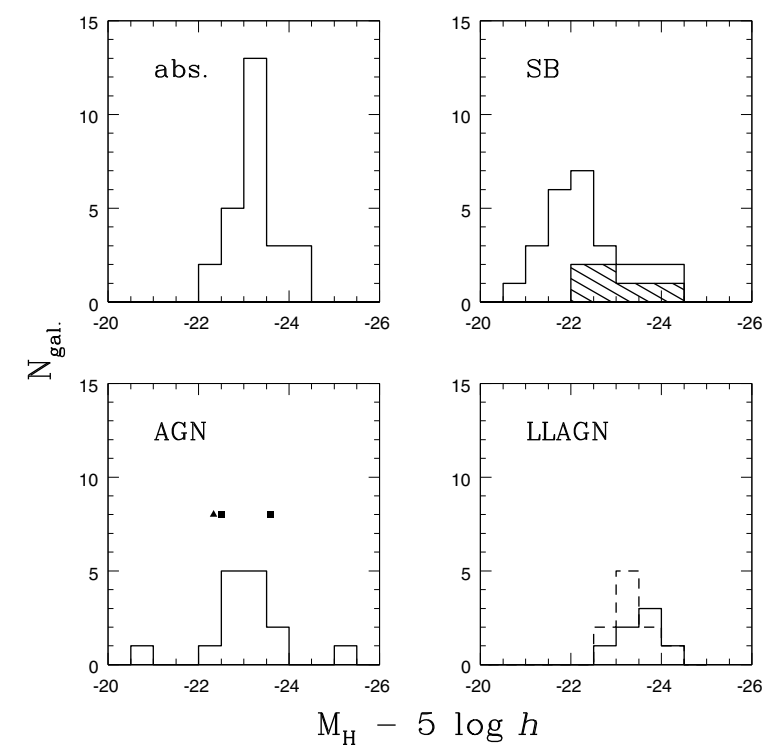

Fig. 10. $M_{H}$ distribution of galaxies with absorption spectra (upper left) and classified as SB (upper right), AGN (lower left) and LLAGN (lower right). The continuous distribution in the upper right panel represents the whole SB population, the dashed histogram 2-line SBs. As in Fig. 8 we have marked in the AGN panel (lower left) the position of the 2 LINERs (squares) and the Sy 2 (triangle) which have not been included in the histogram. In the lower right panel the continuous and dashed line refer respectively to LLAGNs and LLAGN candidates.

while for $r_{\mathrm{p}}>160 \mathrm{~h}^{-1}$ only $2 \mathrm{SBs}$ are found (with an average value of $\mathrm{H}_{\alpha} E W$ of $21.89 \AA$ ). We cannot confirm the anticorrelation between $\mathrm{H}_{\alpha} E W$ and galaxy-galaxy projected distance found by Barton et al. (2000), which is not surprising since our sample is quite different from theirs and we have few (9) galaxy pairs characterized by small $\left(\sim 50 h^{-1} \mathrm{kpc}\right)$ galaxy-galaxy separation. The lack of a clear anticorrelation in our sample might indicate that for bright isolated galaxies in pairs, interaction is at work and is effective up to $160 \mathrm{~h}^{-1} \mathrm{kpc}$.

Figure 10 shows the $M_{H}$ distribution of galaxies in our sample showing absorption lines (upper left) and classified as SB (upper right), AGN (lower left) and LLAGN/LLAGN candidates (lower right, continuous/dashed histogram). The continuous distribution in the upper right panel refers to the whole SB population, the dashed histogram to 2-line SBs. $M_{H}$ has been derived from 2MASS data available for all but 6 galaxies in our sample, which are 3 SBs (UZC-BGP 29B 49A and 74B), 2 absorption line galaxies (UZC-BGP 49B and 58B) and 1 LLAGN (UZC-BGP 26B). At variance with Fig. 8 in which galaxies display rather similarly large $B$ luminosity distributions, Fig. 10 shows that only SBs have a broad $M_{H}$ distribution extending over $4 \mathrm{mag}$; in all the other cases galaxies are concentrated in a 2 mag range. The only exception is AGNs whose distribution, however, appear broad due to the presence of only two galaxies: the faintest and the brightest, in $\mathrm{H}$, of the whole sample. The faintest $\left(M_{H}=-20.91+5 \log h\right)$ is UZC-BGP $24 \mathrm{~B}$, an Sbc galaxy, the brightest $\left(M_{H}=-25.03+5 \log h\right)$ is UZCBGP 79A, an elliptical whose Sy 2 nature we do not confirm due to our imposed emission line $S / N$ threshold. From Fig. 10 we see that while $10 / 26 \mathrm{SB}$ galaxies have $M_{H} \geq-22+5 \log h$, there are no absorption line galaxies fainter than that limit and only 1/18 AGNs. LLAGNs and LLAGN candidates are even brighter as they all show $M_{H}<-22.5+5 \log h$. The upper right panel of Fig. 10 shows that 2-line SBs (dashed histogram) are all found at high $\mathrm{H}$ luminosity and constitute more than half of 
Table 7. The mass of galaxies with different nuclear activity type.

\begin{tabular}{lrrrccccc}
\hline \hline Activity & $N_{\mathrm{T}}$ & $N_{H}$ & $N_{M \text { dyn }}$ & $\begin{array}{c}\Delta M_{\text {dyn }} \\
\left(10^{11} M_{\odot}\right)\end{array}$ & $\begin{array}{c}\text { Med }\left(M_{\text {dyn }}\right) \\
\left(10^{11} M_{\odot}\right)\end{array}$ & $N_{\text {spir. }}$ & $\begin{array}{c}\Delta M_{\text {spir. }} \\
\left(10^{11} M_{\odot}\right)\end{array}$ & $\begin{array}{c}\text { Med }\left(M_{\text {spir. }}\right) \\
\left(10^{11} M_{\odot}\right)\end{array}$ \\
\hline none & 28 & 26 & 23 & {$[1.0: 5.5]$} & 1.9 & 8 & {$[1.0: 3.1]$} & \\
SB & 29 & 26 & 26 & {$[0.3: 4.5]$} & 0.8 & & & \\
AGN & 18 & 18 & 17 & {$[0.2: 3.8]$} & 1.5 & 15 & & \\
LLAGN & 8 & 7 & 5 & {$[1.2: 3.2]$} & 2.1 & 4 & & 2.6 \\
LL & 10 & 10 & 8 & {$[1.1: 6.0]$} & 2.4 & 7 & & 2.2 \\
\hline
\end{tabular}

the whole " $H$ luminous" $\left(M_{H}<-22.5+5 \log h\right)$ SB population. A KS test confirms (99.3 c.l.) the difference between the 4-line SB and 2-line SB $M_{H}$ distribution. The $M_{H}$ distribution of SBs, as a whole, differs significantly from that of absorption line galaxies, AGNs, LLAGNs and LLAGN candidates (KS c.l. $\gg 99.9 \%, 99.7 \%, 99.1 \%$ and $99.9 \%$ respectively). The significance of these differences becomes larger if we exclude from the SB class the 2-line SBs, (in this case we obtain a KS c.l. always $>99.9 \%$ for 4 -lines SB vs. AGN, LLAGN and LLAGN candidates respectively).

The luminosity in $H$ relates to the galaxy mass within the optical radius of disc galaxies $\left(M_{\mathrm{dyn}}\right)$, a quantity that can be derived through the relationship $\log \left(M_{\text {dyn }} / M_{\odot}\right)=\log \left(L_{H} / L_{H \odot}\right)+0.66$ (Gavazzi et al. 1996). Adopting $M_{H \odot}=3.39$ (Allen 1973) we have calculated $M_{\mathrm{dyn}}$ for all the disc galaxies (i.e. ellipticals excluded) in the sample having $H$ magnitude available. The results are summarized in Table 7 where we indicate for each activity type (Col. 1), the total number of galaxies $\left(N_{\mathrm{T}}\right)(\mathrm{Col} .2)$, the number of galaxies $\left(N_{H}\right)$ having $H$ magnitude available (Col. 3) and the number of galaxies $\left(N_{M_{\mathrm{dyn}}}\right)$ for which we have derived the galaxy mass $\left(M_{\mathrm{dyn}}\right),(\mathrm{Col} .4)$. In Col. 5 we give the minimum and maximum value of the mass for each distribution $\left(\Delta M_{\mathrm{dyn}}\right)$ and in Col. 5 the median value of each distribution $\left(\operatorname{med}\left(M_{\text {dyn }}\right)\right)$. With the term none (first row of Col. 1) we indicate galaxies with absorption lines only in their spectrum. Since the nature of S0 galaxies is somewhat questioned and since among them there might be some misclassified ellipticals we have also computed $M_{\text {dyn }}$ for spiral galaxies only. The results, only if different from values reported in Cols. 3-5, are shown in Cols. 6-8 in which we indicate the number of spiral galaxies $\left(N_{\text {spir }}\right)$, minimum and maximum value of the mass $\left(\Delta M_{\text {spir }}\right)$ and median value of the mass distribution (med $\left(M_{\text {spir }}\right)$ ). The exclusion of S0s does not significantly change the results as the mass ranges remain the same in all but the sample of absorption line galaxies, and median values display, consequently, modest changes (only for LLAGNs and LLAGN candidates).

Absorption line galaxies, LLAGNs and LLAGN candidates display higher values of the mass, and SB the lowest, as this is the only population with a median value of the mass distribution below $10^{11} M_{\odot}$. However, if we separate 2-line SBs from 4-line SBs the median value of the first ones increases to $1.3 \times 10^{11} M_{\odot}$ and the mass range narrows (between $7.8 \times 10^{10}$ and $4.4 \times 10^{11} M_{\odot}$ ), while the second ones display a mass range between $2.5 \times 10^{10}$ and $4.5 \times 10^{11} M_{\odot}$ and a median value of $6.5 \times 10^{10} M_{\odot}$. AGNs appear characterized by a wide mass distribution too, which is induced by the presence of an extremely low mass galaxy (UZC-BGP 24B, see also Fig. 10 and relative comments). Exclusion of this galaxy from the AGN sample would raise the minimum mass to $7.9 \times 10^{10}$ (still below the minimum for absorption line galaxies, LLAGNs and LLAGN candidates) and the median value to $1.6 \times 10^{11} M_{\odot}$.

Kauffmann et al. (2003) have shown that AGNs reside only in galaxies with masses $M>10^{10} M_{\odot}$, a value that should be
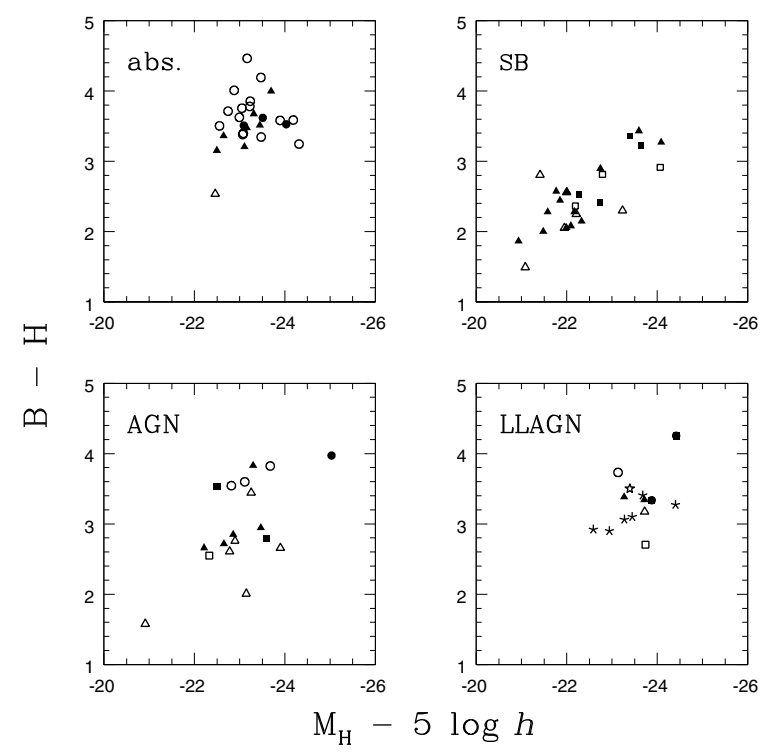

Fig. 11. Color magnitude diagram $\left(B-H\right.$ vs. $\left.M_{H}\right)$ for galaxies with absorption spectra (upper left) and classified as SBs (upper right), AGNs (lower left), LLAGNs and LLAGN candidates (lower right). Filled and empty circles and triangles indicate, in each panel, ellipticals, S0s, early and late spirals. In the SB panel (upper right) the previously defined symbols indicate the morphological classification of 4-line SBs, while filled and empty squares indicate early and late spirals of 2-line SBs. In the AGN panel (lower left) filled and empty squares indicate the 3 galaxies classified respectively as LINERs and Sy 2 . In the LLAGNs/LLAGN candidates panel (lower right) the standard symbols (filled/open circles/triangles) distinguish LLAGN morphological types while we have used filled/open squares to indicate LLAGN candidate ellipticals and SOs and filled/empty stars to indicate early/late spirals.

scaled to $0.7 \times 10^{10} M_{\odot}$ to account for the different values of $H_{0}$ adopted by us and by them. Table 7 shows that, due to the imposed criterion on luminosity (see also Sect. 2), all galaxies in our sample have masses above the Kauffmann et al. (2003) value and we are thus unable to confirm their finding.

Figure 11 illustrates the Color Magnitude (CM) diagram ( $B-H$ vs. $\left.M_{H}\right)$ for galaxies with absorption spectra (upper left) and classified as SB (upper right), AGN (lower left), LLAGN and LLAGN candidates (lower right). We distinguish, for each activity class, different morphological types. Filled and empty circles indicate ellipticals and S0s, while filled and empty triangles stand for early and late spirals. This holds for the absorption line galaxies, 4-line SBs (although this class of objects does not contain early-type galaxies), AGNs and LLAGNs. Concerning 2-line SBs (upper right panel), early and late spirals are indicated as filled and empty squares, while for LLAGN candidates (lower right panel) we have adopted filled and empty squares to indicate $\mathrm{E}$ and $\mathrm{S} 0 \mathrm{~s}$ and filled and empty stars to indicate early and late spirals. In the AGN panel (lower left) empty and filled 
squares indicate the Sy 2 and LINER location (all three are early spirals).

Absorption line galaxies (upper left panel of Fig. 11) occupy a well defined "red-bright" region in the CM diagram, with the exception of UZC-BGP 8A (NGC 800) which is the only late type spiral with an absorption spectrum in our sample. The absence of a trend for absorption line galaxies in the CM diagram must be attributed to the large group of S0s (empty circles) that dominates this population. Early spirals with an absorption spectrum (filled triangles) follow the expected trend and become more $H$ luminous as they become redder ( $B-H$ increasing). SB galaxies (upper right panel) follow a much better defined sequence which holds over a wide range of luminosities. The high proportion of filled vs. empty symbols indicates the dominance of early spirals among this class of objects. The faint luminosity region is mostly occupied by a population of early spirals which, besides being quite faint $\left(M_{H} \geq-22+5 \log h\right)$, are also exceptionally "blue" ( $B-H \sim 2.2)$. As expected (cf. Fig. 9), early and late spirals classified as 2-line SBs (filled and empty squares) occupy the high $H$ luminosity "red" region of the diagram. They too follow the general SB trend. The AGN panel shows the absence of a trend and all but 2 (the brightest and the faintest) AGNs are concentrated in a narrow region which resembles the absorption line galaxy "cloud" but is characterized by a bluer color and a somewhat fainter $H$ luminosity. The AGN "cloud" is made up of two different regions characterized by similar $H$ luminosity and different color. The separation of the two AGN regions occurs at $B-H \sim 3$, which is also the value below which almost no absorption line galaxies are found and where most SB galaxies reside. Also LLAGN and all but one LLAGN candidates occupy a well defined region in the CM diagram which is brighter than the ones occupied by absorption line galaxies and AGNs and has a color in between the two. Figure 11 indicates that absorption line galaxies, SBs, AGNs and LLAGNs occupy rather distinct regions in this $\mathrm{CM}$ diagram so that it would possible to distinguish SB from absorption line galaxies on the basis of their location in this diagram. The AGN location is in between the previous two, but is almost completely separated from LLAGN and LLAGN candidates. This is an interesting finding that should be verified on galaxy samples in different environments.

\section{Nuclear activity in pairs showing interaction}

There are cases in which galaxy interaction produces "visible" effects such as morphological distortions, bridges and tidal tails. However, the detection of those features depends on the sensitivity of the instrument to low surface brightness structures and is thus strongly affected by seeing, depth and redshift. Moreover morphological distortions are short lived phenomena (100 Myr) and tidal tails appear only in prograde disk encounters (Toomre $\&$ Toomre 1972). Thus a selection of systems based on the presence of "visible" signs of interaction would lead to a fraction of interacting pairs being missed. However, pairs showing interaction can be used to test the pair sample as a whole, as differences that might be present in the content and type of nuclear activity between these systems and the whole pair sample could bring into question the interaction status of galaxy pairs.

Careful inspection of DSS images of all pairs in our sample allowed us to identify 9 pairs in which interaction is detected between the members. These pairs are listed in Table 8, where we give, for each of them, identifier (Col. 1), pair/galaxy morphology (Col. 2; as in Tables 4-6 galaxy morphology of the active member is underlined in $\mathrm{E}+\mathrm{S}$ pairs), nuclear
Table 8. UZC-BGPs showing interaction between members.

\begin{tabular}{|c|c|c|c|c|c|}
\hline \multirow[t]{2}{*}{ UZC-BGP } & \multirow[t]{2}{*}{ Pair type } & \multicolumn{2}{|c|}{ Activity type } & \multicolumn{2}{|c|}{$\mathrm{H}_{\alpha} E W$} \\
\hline & & A & B & A & B \\
\hline 8 & $\mathrm{~S}+\mathrm{S}$ & - & SB & - & 12.46 \\
\hline 14 & $\mathrm{E}+\underline{S}$ & SB & - & 74.51 & - \\
\hline 21 & $\mathrm{E}+\bar{S}$ & - & LLAGN & - & 0.31 \\
\hline 49 & $\mathrm{~S}+\overline{\mathrm{S}}$ & SB & - & 20.51 & - \\
\hline 59 & $\mathrm{~S}+\mathrm{S}$ & LL & AGN & - & 9.39 \\
\hline 65 & $\mathrm{E}+S$ & SB & - & 43.47 & - \\
\hline 71 & $\mathrm{~S}+\overline{\mathrm{S}}$ & LL & AGN & - & 1.84 \\
\hline 74 & $\mathrm{~S}+\mathrm{S}$ & SB & SB & 58.94 & 57.30 \\
\hline 83 & $\mathrm{~S}+\mathrm{S}$ & Sy2 & SB & 117.43 & 85.01 \\
\hline
\end{tabular}

Table 9. UZC-BGP galaxies interacting with fainter companions.

\begin{tabular}{rclrl}
\hline \hline UZC-BGP & Pair type & Activity type & $\mathrm{H}_{\alpha} E W$ & Companion \\
\hline 4B & $\mathrm{E}+\underline{S}$ & LLAGN & 0.34 & IC 1559 \\
5B & $\underline{E}+\overline{\mathrm{S}}$ & - & - & NGC 197 \\
9B & $\overline{\mathrm{S}}+\mathrm{S}$ & SB & 11.22 & NGC 876 \\
10A & $\underline{E}+\mathrm{S}$ & LLAGN & 1.66 & NGC 997N \\
$16 \mathrm{~A}$ & $\underline{E}+\mathrm{S}$ & - & - & NGC 1588 \\
20A & $\overline{\mathrm{E}}+\underline{S}$ & SB & 76.86 & NGC 2744S \\
20B & $\underline{E}+\bar{S}$ & LLAGN & 1.02 & NGC 2751 \\
26B & $\underline{E}+\mathrm{S}$ & LLAGN & 0.47 & IC 590 E \\
28A & $\overline{\mathrm{S}}+\mathrm{S}$ & LINER & 11.42 & NGC 3303 N \\
\hline
\end{tabular}

activity type (Col. 3 ; - indicates absence of nuclear activity) and $\mathrm{H}_{\alpha} E W$ (Col. 4; only if the galaxy is active).

Among the 48 galaxy pairs of our sample only 9 (listed in Table 8) are characterized by signs of interaction between their members. Table 8 shows that this happens more frequently in $\mathrm{S}+\mathrm{S}(6 / 23)$ than in $\mathrm{E}+\mathrm{S}(3 / 19)$ pairs and that in these last cases interaction is never able to induce emission activity in the early type member of the pair. On the other hand interaction does not appear to be able to induce nuclear activity in 2 galaxies belonging to the $\mathrm{S}+\mathrm{S}$ pairs either. All kinds of activities are found in pairs listed in Table 8; visible interaction thus does not appear to be responsible for activation of a particular kind of nuclear activity. In Table 8 there are 7 SB galaxies, 3 AGNs (Sy 2 included), 1 LLAGN, 2 LLAGN candidates and 5 galaxies with absorption spectra. This implies fractions of activity type per galaxy of $39 \%, 17 \%, 6 \%, 11 \%$ and $28 \%$ which are remarkably similar to the ones for the whole sample $(30 \%, 19 \%, 8 \%, 11 \%$ and $30 \%)$. Five of the SBs in Table 8 are 4-line SBs, two (UZC-BGP 8A and 49A) are 2-line SBs. Thus also the fraction of 2 to 4-line $\mathrm{SBs}$ in these pairs agrees remarkably well with the fraction in the whole sample $(8 / 21)$. From Col. 5 of Table 8 we can derive the median value of $\mathrm{H}_{\alpha} E W$ of 4-line and 2-line SB respectively $58.94 \AA$ and $16.49 \AA$, again very similar to the ones derived for the whole sample (cf. Sect. 4). The similarity in terms of amount, type and characteristics of the nuclear activity between these 9 pairs and the whole sample supports the hypothesis that galaxygalaxy interaction between bright galaxies in isolated pairs is at work and effective.

Since UZC-BGP is a volume-limited sample, interaction may even occur between one pair member and a faint companion that has gone undetected either because of the UZC catalog magnitude limit $\left(m_{\mathrm{Zw}} \leq 15.5\right)$ or the UZC-BGP luminosity limit $\left(M_{\mathrm{Zw}} \leq-18.9+5 \log h\right)$. This happens for 9 galaxies, belonging to 8 distinct pairs, which we list in Table 9. For each galaxy we give identifier (Col. 1), pair/galaxy morphology (Col. 2; as in Table 8 galaxy morphology is underlined in case of $\mathrm{E}+\mathrm{S}$ ), activity type (Col. 3 ; - means no activity), $\mathrm{H}_{\alpha} E W$, identificator of 
Table 10. UZC-BGPs with absorption line spectra in both members.

\begin{tabular}{rcl}
\hline \hline UZC-BGP & Pair type & Interaction \\
\hline 23 & E+E & A with NGC 2988 \\
31 & S+S & no \\
35 & E+E & A with B \\
58 & E+E & A with B \\
63 & E+E & no \\
67 & E+S & no \\
76 & S+S & no \\
\hline
\end{tabular}

the companion (Col. 5). Among these faint companions only two (NGC 1588 and NGC 2751) interacting respectively with UZCBGP 16A and 20B have failed the luminosity limit of UZC-BGP, in all other cases companions were too faint to be included in the UZC catalog.

At variance with Table 8, Table 9 shows that most (7/9) of the galaxies interacting with fainter companions belong to $\mathrm{E}+\mathrm{S}$ pairs. Five galaxies in Table 9 are early-type, two of them (UZCBGP $5 \mathrm{~B}$ and 16A) display no activity and the remaining three (UZC-BGP 10A, 20B and 26B) show LLAGN activity. This last one is the most frequent kind of activity of galaxies in Table 9. The fraction of LLAGNs among the galaxies of Table 9 is $44 \%$ (to be compared with $8 \%$ in the whole sample). The excess of LLAGNs could indicate minor galaxy interaction (Woods \& Geller 2007) as a driving mechanism for this kind of activity. Interestingly, the interacting LLAGNs of Table 9 are all found in $\mathrm{E}+\mathrm{S}$ pairs which we expected (cf. Fig. 3 and discussion) to be the bright core of a looser structure. The presence of a fainter close companion confirms both our expectation and previous finding (Coziol et al. 1998, 2000; Martinez et al. 2006) that LLAGN is the most common kind of activity in Compact Groups. Further investigation is needed since no detailed analysis concerning the link between LLAGN activity and interaction has been carried out so far.

Finally, if interaction plays a major role in activating nuclear activity, passive galaxies in galaxy pairs should not display evident interaction. There are 7 pairs in our sample with both members passive. They are listed in Table 10 where we give, the pair identifier (Col. 1), morphology (Col. 2) and, in Col. 3, we indicate if interaction occurs between pair members or with a faint companion. Only one galaxy (UZC-BGP 23A) interacts with a faint companion (NGC 2988, which was not included in the UZC catalog). The sample is dominated by $\mathrm{E}+\mathrm{E}$ pairs $(4 / 7)$, 3 of which show interaction patterns, the remaining $2 \mathrm{~S}+\mathrm{S}$ and $\mathrm{E}+\mathrm{S}$ pairs do not show interaction at all. There is interaction and no activity only in the $3 \mathrm{E}+\mathrm{E}$ pairs, which is somewhat encouraging for the interaction-activity scenario as galaxy interaction will produce larger effects in gas rich than in gas poor galaxies, while the $2 \mathrm{~S}+\mathrm{S}$ pairs are characterized by large values (159 and $183 h^{-1} \mathrm{kpc}$ ) of $r_{\mathrm{p}}$ (cf. Fig. 3, upper middle panel) in our sample. This confirms our previous finding on $\mathrm{H}_{\alpha} E W$ and suggest that in the UZC-BGP sample interaction is at work and effective up to $160 h^{-1} \mathrm{kpc}$.

\section{Conclusions}

To investigate the role of galaxy interaction on nuclear activity we performed a detailed spectroscopical analysis on 48 galaxy pairs which represent more than half of the whole UZC-BGP sample and have an excellent morphological match with it.
We found an extremely large fraction of emission line galaxies in our sample, particularly among early (84\%) and late (95\%) spirals.

Classification and analysis of spectral activity, performed by means of standard diagnostic diagrams, allowed us to show that SB is the most frequent (30\% of galaxies) kind of activity in our sample. It occurs exclusively in spiral galaxies with a frequency of the SB phenomenon among spirals of $45 \%$. The blue luminosity distribution of these SB is not particularly high as $67 \%$ have $M_{B}>-20+5 \log h$.

While SB are preferentially found in $\mathrm{S}+\mathrm{S}$ pairs, AGN are almost equally found in $\mathrm{S}+\mathrm{S}$ and in $\mathrm{E}+\mathrm{S}$ pairs although in most cases $(82 \%)$ this kind of activity is displayed by a spiral galaxy. AGN in our sample show rather advanced morphological distribution characterized by a high blue luminosity. The fraction of AGNs in late spirals is $35 \%$ and late spirals hosting AGNs have an average $M_{B}=-20.4+5 \log h$.

SBs display enhanced $\mathrm{H}_{\alpha} E W$, an effect which relates to star formation and might thus be related to pair environment. Star formation is intense in half of the SB galaxies in our sample. Intense-SBs have galaxy-galaxy separations up to $160 \mathrm{~h}^{-1} \mathrm{kpc}$ implying that interaction may be effective in isolated pairs of bright galaxies up to that distance.

Absorption line galaxies, SBs, AGNs and LLAGNs (candidates included) occupy rather distinct locations in the $B-H$ vs. $M_{H}$ diagram, a characteristics which reflects the different distribution in $B$ and $H$ luminosity of each sample. Galaxy masses, estimated using the $H$ luminosity, are high for absorption line galaxies and LLAGNs (as a whole), low for SBs and "intermediate" for AGNs

All LLAGNs reside in E+S and are equally distributed between early type galaxies and spirals. We have shown that half of them are hosted in galaxies displaying signs of interaction with fainter companions, which suggests that minor interaction might be a driving mechanism in some fraction of LLAGNs. LLAGN has been claimed to be a heterogenous class of objects: our previous finding concerning only half of the whole LLAGN population, coupled with the quite different behaviour in terms of blue luminosity and morphological content of LLAGN candidates, appears to confirm that claim.

Acknowledgements. This work was supported by MIUR. P.F. acknowledges financial support from the contract ASI-INAF I/023/05/0. S.M. acknowledges a fellowship by INAF-OAB. This research has made use of the NASA/IPAC Extragalactic Database (NED) and of the Hyperleda Database (http://leda. univ-lyon $1 . \mathrm{fr} /$ ). We thank the anonymous referee whose comments and criticism greatly improved the paper.

\section{References}

Allen, C. 1973, Astrophysical Quantities (London: The Athlone Press)

Alonso, M. S., Tissera, P. B., Colwell, G., \& Lambas, D. 2004, MNRAS, 352, 1081

Bahcall, N. A., Lubin, J. M., \& Dorman, V. 1995, ApJ, 447, L81

Baldwin, J. A., Phillips, M. M., \& Terlevich, R. 1981, PASP, 93, 5

Barnes, J. E., \& Hernquist, L. E. 1991, ApJ, 370, 65

Barton, E. J., Geller, M. J., \& Kenyon, S. J. 2000, ApJ, 530, 660

Borne, K. D., Bushouse, H., Colina, L., et al. 1999, Ap\&SS, 266, 137

Bundy, K., Fukugita, M., Ellis, R. S., Kodama, T., \& Conselice, C. J. 2000, ApJ, 601,123

Carlberg, R. G., Pritchet, C. J., \& Infante, L. 1994, ApJ, 435, 540

Coziol, R., Ribeito, A. L. B., de Carvalho, R. R., \& Capelato, H. V. 1998, ApJ, 493, 563

Cuesta-Bolao, M. J., \& Serna, A. 2003, A\&A, 405, 917

Dahari, O. 1985, ApJS, 57, 643

De Robertis, M. M., Hayhoe, K., \& Yee, H. K. C. 1998, ApJS, 115, 163

de Vaucouleurs, G., \& de Vaucouleurs, A. 1964, Reference Catalogue of Bright Galaxies (RC1), University of Texas Monography in Astronomy No.1 (University of Texas Press) 
Donzelli, C., \& Pastoriza, M. G. 1997, ApJS, 111, 181

Dubinski, J., Mihos, J. C., \& Hernquist, L. 1996, ApJ, 462, 576

Falco, E. E., Kurtz, M. J., Geller, M. J., et al. 1999, PASP, 111, 438

Focardi, P., \& Kelm, B. 2002, A\&A, 391, 35

Focardi, P., Zitelli, V., Marinoni, S., \& Kelm, B. 2006, A\&A, 456, 467 (Paper I)

Fuentes-Williams, T., \& Stocke, J. T. 1988, AJ, 96, 1235

Gavazzi, G., Pierini, D., \& Boselli, A. 1996, A\&A, 312, 397

Gonçalves, A. C., Véron-Cetty, M.-P., \& Véron, P. 1999, A\&AS, 135, 437

Gottlober, S., Klypin, A., \& Kravstov, A. V. 2001, ApJ, 546, 223

Governato, F., Gardner, J. P., Stadel, J., Quinn, T., \& Lake, G. 1999, MNRAS, 307, 949

Ho, L. C., Filippenko, A. V., \& Sargent, W. L. W. 1994, IAU Symp., 159, 275

Ho, L. C., Filippenko, A. V., \& Sargent, W. L. W. 1997, ApJ, 487, 568

Karachentsev, I. D. 1972, in Catalogue of Isolated Pairs in the Northern Emisphere, Comm. Spec. Ap. Obs., 7, 1 (KPG)

Kauffmann, G., Heckman, T. M., Tremonti, C., et al. 2003, MNRAS, 346, 1055

Keel, W. C. 1993 , AJ, 106, 1771

Keel, W. C. 1996, AJ, 111, 696

Keel, W. C., Kennicutt, R., Hummel, E., \& van der Hulst, J. 1985, AJ, 90, 708

Kelm, B., \& Focardi, P. 2004, A\&A, 418, 937

Kelm, B., Focardi, P., \& Palumbo, G. G. C. 1998, A\&A, 335, 912

Kelm, B., Focardi, P., \& Zitelli, V. 2004, A\&A, 418, 25

Kelm, B., Focardi, P., \& Sorrentino, G. 2005, A\&A, 442, 117

Kennicutt, R. C. Jr., \& Keel, W. C. 1984, ApJ, 279, L5

Kennicutt, R. C. Jr., \& Kent, S. M. 1983, AJ, 88, 1094

Kennicutt, R. C. Jr., Keel, W. C., van der Hulst, J. M., \& Hummel, E. 1987, AJ, 93, 1011

Kennicutt, R. C. Jr., Tamblin, P., \& Congdon, C. E. 1994, ApJ, 435, 22

Kewley, L., Dopita, M., Sutherland, R., Heisler, C., \& Trevena, J. 2001, ApJ, 556,121
Kewley, L. J., Groves, B., Kauffmann, G., \& Heckman, T. 2006, MNRAS, 372, 961

Larson, R. B., \& Tinsley, B. M. 1978, ApJ, 219, 46

Lauberts, A., \& Valentijn, E. 1989, The Surface Photometry Catalogue of the ESO-Uppsala Galaxies, ESO, Garching bei Munchen (ESO-LV)

Le Fevre, O., Abraham, R., Lilly, S., et al. 2000, MNRAS, 311, 565

MacKenty, J. W. 1989, ApJ, 343, 125

Martinez, M. A., del Olmo, A., Perea, J., \& Coziol, R. 2006, in Groups of Galaxies in the Nearby Universe, ESO-workshop [arXiv: astro-ph/0611098]

Mihos, J., \& Hernquist, L. 1996, ApJ, 464, 641

Miller, C. J., Nichol, R. C., Gomez, P. L., Hopkins, A. M., \& Bernardi, M. 2003, ApJ, 597, 142

Noguchi, M. 1988, A\&A, 203, 259

Patton, D. R., Carlberg, R. G., Marzke, R. O., et al. 2000, ApJ, 536, 153

Paturel, G., Petit, C., Prugniel, Ph., et al. 2003, A\&A, 412, 45

Rafanelli, P., Violato, M., \& Baruffolo, A. 1995, AJ, 109, 1546

Reduzzi, L., \& Rampazzo, R. 1995, ApL\&C, 30, 1 (RR)

Sanders, D. B., \& Mirabel, I. F. 1996, ARA\&A, 34, 749

Sanders, D. B., Soifer, B. T., Elias, J. H., et al. 1988, ApJ, 325, 74

Schmitt, H. R. 2001, AJ, 122, 2243

Toomre, A., \& Toomre, J. 1972, ApJ, 178, 623

Veilleux, S. 2002, in AGN Surveys, IAU Coll. 184, ed. R. F. Green, E. Ye. Khachikian, \& D. B. Sanders, ASP Conf. Proc., 284, 111

Veilleux, S., \& Osterbrock, D. E. 1987, ApJS, 63, 295

Veilleux, S., Kim, D. C., Sanders, D. B., Mazzarella, J. M., \& Soifer, B. T. 1995, ApJS, 98, 171

Véron, P., Gonçalves, A. C., \& Véron-Cetty, M. P. 1997, A\&A, 319, 52

Woods, D. F., \& Geller, M. 2007, AJ 134, 527

Zaritsky, D., Smith, R., Frenk, C., \& White, S. D. M. 1997, ApJ, 478, 39

Zepf, S. E., \& Koo, D. C. 1989, ApJ, 337, 34 